\title{
The continuous pollution routing problem
}

Yiyong $\mathrm{Xiao}^{\mathrm{a}}$, Xiaorong Zuo ${ }^{\mathrm{a}}$, Jiaoying Huang ${ }^{\mathrm{a}}$, Abdullah Konak ${ }^{\mathrm{b}}$, Yuchun $\mathrm{Xu}^{\mathrm{c}}$

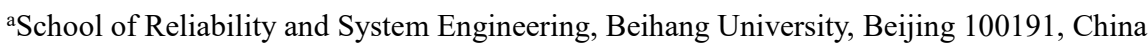

bInformation Sciences and Technology, Penn State Berks, Tulpehocken Road, P.O. Box 7009, Reading, PA 19610-6009, United States

'School of Engineering \& Applied Science, Aston University, Birmingham, B4 7ET, United Kingdom

\begin{abstract}
In this paper, we presented an $\varepsilon$-accurate approach to conduct a continuous optimization on the pollution routing problem (PRP). First, we developed an $\varepsilon$-accurate inner polyhedral approximation method for the nonlinear relation between the travel time and travel speed. The approximation error was controlled within the limit of a given parameter $\varepsilon$, which could be as low as $0.01 \%$ in our experiments. Second, we developed two $\varepsilon$-accurate methods for the nonlinear fuel consumption rate (FCR) function of a fossil fuel-powered vehicle while ensuring the approximation error to be within the same parameter $\varepsilon$. Based on these linearization methods, we proposed an $\varepsilon$-accurate mathematical linear programming model for the continuous PRP ( $\varepsilon$-CPRP for short), in which decision variables such as driving speeds, travel times, arrival/departure/waiting times, vehicle loads, and FCRs were all optimized concurrently on their continuous domains. A theoretical analysis is provided to confirm that the solutions of $\varepsilon$-CPRP are feasible and controlled within the predefined limit. The proposed $\varepsilon$-CPRP model is rigorously tested on well-known benchmark PRP instances in the literature, and has solved PRP instances optimally with up to 25 customers within reasonable CPU times. New optimal solutions of many PRP instances were reported for the first time in the experiments.
\end{abstract}

Keywords: vehicle routing problem; emission reduction; continuous optimization; convex programming

\section{Introduction}

According to the report published by the International Energy Agency (IEA, 2016a), the transportation sector was the second largest contributor to $\mathrm{CO}_{2}$ emissions, a well-known greenhouse gas accounting for $23 \%$ of the global $\mathrm{CO}_{2}$ emissions in 2014, and road transportation was responsible for almost three-quarters of the total emissions resulting from transportation activities. Overall, the transportation system of the modern world is still heavily dependent on burning fossil fuels (gasoline, diesel, petroleum, and natural gas), a major source of $\mathrm{CO}_{2}$ emissions from human activities. For example, fossil fuels accounted for $91 \%$ of the total energy consumed in the USA transportation sector in 2016 (EIA, 2017). Although alternative energy vehicles, such as electric, hydrogen, and solar vehicles, are promising options for reducing $\mathrm{CO}_{2}$ emissions in transportation, they have not been widely applied owing to various reasons such as high investment costs, short travel ranges, and lack of recharging stations. Even in China, the world's largest electric vehicle market, electric vehicles comprised only $1 \%$ of the market in 2015, and globally, electric vehicles account for only $0.9 \%$ (IEA, 2016b). Alternative energy vehicles are still facing some technical and economic challenges today.

Another effective way of reducing vehicle emissions is to improve the efficiency of transportation systems through better operational strategies. The pollution routing problem (PRP) initialized by Bektas and Laporte (2011) is a widely studied optimization problem that involves balancing the operational/monetary cost and environmental

\footnotetext{
${ }^{*}$ Corresponding Author:

Email addresses: xiaoyiyong@buaa.edu.cn (Y., Xiao), zuoxiaorong710@163.com (X., Zuo), huangjy@buaa.edu.cn (J., Huang), auk3@psu.edu (A., Konak),y.xu16@Aston.ac.uk (Y., Xu)
} 
cost of logistic companies that serve their customers with fossil fuel-powered vehicles. However, owing to nonlinear relationships existing in PRP, such as the time-speed relation and the fuel consumption rate (FCR) function, it is difficult to formulate PRP as a continuous linear model. Bektas and Laporte (2011) first adopted a discretization strategy over the travel speeds, by which the vehicles can only travel at discrete speeds selected from a prespecified speed set. This discretization strategy was also used by other variants/extensions of PRP in the literature, including the time-dependent PRP (TD-PRP) by Franceschetti et al. (2013), the bi-objective PRP by Demir et al. (2014b), and the heterogeneous PRP by Koc et al. (2014). However, the discretized speeds in PRP lead to discretized travel times and discretized FCRs in the solution, which increase the combinational complexity and result in sub-optimal solutions.

This study extended the discrete PRP of Bektas and Laporte (2011) to a continuous case by taking the travel speed as a continuous decision variable, such that all related scheduling variables including travel time, load flow, FCR, and departing/arrival/waiting/ times are all treated as continuous decision variables. Therefore, we called this problem as a continuous PRP (CPRP). We developed an $\varepsilon$-accurate mathematical linear programming model for the CPRP ( $\varepsilon$-CPRP for short), in which all variables are optimized synchronously within their continuous domains. All nonlinear components in $\varepsilon$-CPRP are linearized by a unified parameter $\varepsilon$ to control the approximation error within the range of $\varepsilon \%$. Thus, the proposed $\varepsilon$-CPRP model is expected to obtain truly optimized solutions. As demonstrated in our computational experiments, the parameter $\varepsilon$ can be set as low as $0.01 \%$ without increasing the computational burden significantly. In addition, we also prove that the gap between the solution found by the $\varepsilon$-CPRP model and the optimal one is within $3 \varepsilon \%$. Therefore, the solution can be considered as optimal from a practical point of view. More importantly, the proposed linearization approach does not require additional binary variables to the model, which is an important contribution of the paper. Therefore, it is computationally efficient.

The rest of the paper is organized as follows. In Section 2, the related literature review is provided. In Section 3, a linearization method is provided for the nonlinear relationship between the travel time and travel speed. In Section 4, two linearization methods for the nonlinear FCR function are provided. Based on these methods, we propose the $\varepsilon$-CPRP model in Section 5 and prove its feasibility and optimality. Section 6 presents the computational experiments conducted, and finally, we conclude this research in Section 7.

\section{Related literature reviews}

In recent years, green-oriented vehicle routing problems (VRPs), which incorporate environmental concerns such as pollution mitigation, emission reduction, and environmental sustainability into VRPs, have attracted a significant level of interest from operations research professionals (see surveys of Lin et al. (2014) and Demir et al. (2014a)). Whereas conventional VRPs aim to optimize vehicle routes typically by minimizing a single monetary cost function, green-oriented VRPs consider both the monetary costs and the environmental impacts and try to optimize both of them together. Because the main contribution of the paper is about efficient modeling of green-oriented VRPs, with the extensive green VRP literature, we primarily focus on the papers related to modeling of the problem. There are a number of optimization models existing in the literature, which generally can be classified into four categories: (1) models minimizing energy/fuel or $\mathrm{CO}_{2}$ emissions through vehicle payload optimization, such as the energy-minimizing VRP (EMVRP) model by Kara et al. (2007, 2008), FCR-considered Capacitated VRP model by Xiao et al. (2012), and cumulative VRP model by Gaur et al. (2013); (2) models minimizing $\mathrm{CO}_{2}$ emissions by 
optimizing vehicle speed, such as the emission-oriented time-dependent VRPs (TD-VRPs) by Figliozzi (2010), Kuo (2010), and Jabali et al. (2012); (3) the PRP model by Bektas and Laporte (2011), which minimizes driver and fuel-related costs by optimizing both speed and load simultaneously; and (4) models minimizing $\mathrm{CO}_{2}$ emissions by avoiding traffic congestions and by load optimization, such as the green vehicle routing and scheduling problem (GVRSP) by Xiao and Konak (2015, 2016, 2017).

Kara et al. (2007) first proposed the EMVRP, which aims to minimize the total energy consumed along vehicle routes instead of the conventional objective of minimizing total travel distance. Kara et al. (2008) and Gaur et al. (2013) studied the cumulative VRP (Cum-VRP), which minimizes the total fuel consumption of vehicles in a goods collection scenario, where empty vehicles start from a depot to pick up goods from nodes and loaded vehicles are allowed to offload goods at the depot multiple times. Figliozzi (2010) built a post-optimization model for the solution obtained by a TD-VRP model by which the departure times of vehicles can be optimized for reducing the total emissions. Kuo (2010) first modeled the TD-VRP with a fuel consumption objective in a time-dependent traffic environment, in which the fuel consumption in each traveled arc is considered as a function of vehicle departure time and load. Xiao et al. (2012) extended the CVRP by considering an FCR expressed as a function of the vehicle load. Jabali et al. (2012) studied the emission-based TD-VRP with an objective function including both travel time costs and fuel/emission costs. Zhang et al. (2015) proposed a model for a low carbon routing problem that considers a similar scenario of the Cum-VRP by Kara et al. (2008) and Gaur et al. (2013) with a simplified way of calculating the fuel consumption of a vehicle traveling at a specified speed. Xiao and Konak $(2015,2016,2017)$ studied a GVRSP that involves selecting optimal vehicle routes and schedules to minimize the total $\mathrm{CO}_{2}$ emissions of a fleet of heterogeneous vehicles in situations where time-varying traffic congestions exist. The main difference of GVRSP from PRP is that the former assumes that the vehicle travel speed is determined by the average traffic flows whereas the latter one considers the travel speed as a decision variable.

Erdogan and Miller-Hooks (2012) proposed another version of the green VRP (shortened as G-VRP to differentiate it from GVRSP) for alternative fuel-powered vehicles in a service area with limited refueling infrastructure. In G-VRP, a vehicle needs to visit refueling stations during its tour because the vehicle maximum travel range is limited and the refueling stations are rare and sporadically available. Because the G-VRP of Erdogan and Miller-Hooks (2012) involves only eco-friendly vehicles such as electric and alternative fuel-powered vehicles, their objective function is not about the fuel consumption or $\mathrm{CO}_{2}$ emissions but the conventional total distance. Schneider et al. (2014) extended the G-VRP by considering a fleet of electric vehicles with time windows, recharging at stations, and limited vehicle load capacities. Koç and Karaoglan (2016) proposed a simulated annealing heuristic based on the exact branch-and-cut algorithm for the G-VRP. Leggieri and Haouari (2017) addressed the G-VRP with time duration limits and energy consumption constraints and used the reformulation-linearization technique to linearize the nonlinear formulation.

Bektas and Laporte (2011) first introduced the comprehensive modal emission model (CMEM) of Barth et al. (2005) and Barth and Boriboonsomsin (2008) into the VRP with time windows (VRPTW) so that the FCR of a vehicle can be calculated dynamically according to its travel speed and payload. Therefore, they extended the classical VRPTW to PRP with a comprehensive objective function that includes both fuel-related expenses (e.g., fuel consumption and emission tax) and travel time related costs such as driver's wages. Because the $\varepsilon$-CPRP 
proposed in this study is closely related to PRP, we mainly focus on the works related to PRP in the rest of the literature review.

Demir et al. (2012) and Kramer et al. (2015a, 2015b) studied and proposed solution approaches to the discrete PRP model of Bektas and Laporte (2011). Demir et al. (2012) developed a two-stage algorithm to solve large-sized PRP cases and provided a set of benchmark PRP instances based on the geographical locations of cities in the UK. This two-stage algorithm first uses an adaptive large neighborhood search (ALNS) heuristic to find a solution by treating PRP as a conventional VRPTW with fixed travel speeds and then determines the optimal travel speed for each selected arc of the routes in the second stage. In this approach, the travel speed is selected from a set of discrete values. Kramer et al. (2015a, 2015b) proposed a hybrid of the metaheuristic (an iterated local search heuristic) and a speed optimization algorithm (an exact procedure) for PRP and provided two sets of new benchmark PRP instances.

Franceschetti et al. (2013) extended PRP to TD-PRP by dividing the planning horizon into three periods: (1) a rush-hour period in which traffic congestion is heavy and vehicles have to travel at low speeds, (2) a free-flow period in which vehicles can travel freely at speeds between the lower and upper limits, and (3) a transition period in which the traffic condition is linearly transformed from the rush-hour period to the free-flow period. A similar approach to modeling the time-varying traffic congestion can also be found in Jabali et al. (2012). Franceschetti et al. (2017) developed a metaheuristic for the TD-PRP using an enhanced ALNS algorithm with a departure time and speed optimization procedure.

Other variants and extensions of PRP in the literature include the bi-objective PRP by Demir et al. (2014b), heterogeneous PRP by Koc et al. (2014), time window pickup-delivery PRP (TWPDPRP) by Tajik et al. (2014), practical PRP (PPRP) by Suzuki (2016), mixed-integer convex programming (MICP) model for PRP by Fukasawa et al. (2016), and the robust PRP by Eshtehadi et al. (2017).

Demir et al. (2014b) formulated PRP as a bi-objective problem comprising the fuel consumption objective and the driving time objective. They also provided an enhanced ALNS algorithm with a speed optimization procedure to discover Pareto optimal solutions to a problem. Koc et al. (2014) extended PRP by considering a fleet of heterogeneous vehicles and minimizing the sum of vehicle's fixed costs and routing costs. They provided a hybrid evolution algorithm as the solution approach, which is based on the framework of a genetic algorithm combined with a post-optimization procedure for determining speeds. Tajik et al. (2014) studied the TWPDPRP, in which simultaneous pickup (to the depot) and delivery (from the depot) services are considered with time window requirements. The objective function of TWPDPRP includes driver costs and fuel-related expenses, in which several practical factors, such as the physical condition of roads (i.e., the road friction and road slope), weights and loads of vehicles, surface and air friction, and acceleration and deceleration, are considered to affect the fuel consumption. The main difference between TWPDPRP and PRP is that the former treats the travel speed on each arc as a previously known constant, whereas the latter treats it as a decision variable. Suzuki (2016) proposed the PPRP model, which includes fewer but more practical factors that affect the fuel consumption significantly. In the PPRP model, the "payload" along an arc is considered as an essential factor for fuel consumption, and the travel speed, gradient, and traffic congestion factors are also important but treated as constant parameters associated to each arc. Fukasawa et al. (2016) employed a disjunctive convex programming approach to model PRP as an MICP 
with continuous speed. To our best knowledge, this is the first time for PRP to be modeled with continuous speed variables. Saka et al. (2017) studied the heterogeneous PRP with continuous speed optimization. However, their model contains nonlinear components both in the objective function and in the constraints. Eshtehadi et al. (2017) proposed the robust PRP model, which minimizes the worst-case of fuel consumption for the scenario with demand uncertainty. Dabia et al. (2017) studied a variant of PRP in which the travel speeds over all arcs of a route were assumed to be the same. A summary of the models reviewed above is provided in Table 1.

Table 1. Summary of fuel/emission optimization models in the literature

\begin{tabular}{|c|c|c|c|c|c|c|c|}
\hline & \multirow[b]{2}{*}{ Models } & \multirow[b]{2}{*}{ Objectives to minimize } & \multicolumn{4}{|c|}{ Decision variables } & \multirow[b]{2}{*}{ Sources } \\
\hline & & & Routes & Load & Speed & Dep. T. & \\
\hline 1 & Energy-minimize VRP & Energy consumption & $\sqrt{ }$ & $\sqrt{ }$ & & & Kara et al. (2007) \\
\hline 2 & Cumulative VRPs & Fuel consumption & $\sqrt{ }$ & $\sqrt{ }$ & & & Kara et al. (2008), Gaur et al. (2013) \\
\hline 3 & FCR-considered CVRP & Fuel consumption & $\sqrt{ }$ & $\sqrt{ }$ & & & Xiao et al. (2012) \\
\hline 4 & Green VRP & $\mathrm{CO}_{2}$ emissions & $\sqrt{ }$ & $\sqrt{ }$ & & $\sqrt{ }$ & Figliozzi (2010) \\
\hline 5 & Time-dependent VRP & Fuel consumption & $\sqrt{ }$ & $\sqrt{ }$ & & $\sqrt{ }$ & Kuo (2010) \\
\hline 6 & $\begin{array}{l}\text { Emission-based } \\
\text { Time-dependent VRP }\end{array}$ & $\begin{array}{l}\text { Travel time costs and } \\
\text { fuel/emission costs }\end{array}$ & $\sqrt{ }$ & & $\sqrt{ }$ & & Jabali et al. (2012) \\
\hline 7 & Green VRP (G-VRP) & Total distance & $\sqrt{ }$ & & & & Erdogan and Miller-Hooks (2012) \\
\hline 8 & $\begin{array}{l}\text { Pollution routing problem } \\
(\mathrm{PRP})\end{array}$ & Total time and total fuel & $\sqrt{ }$ & $\sqrt{ }$ & $\sqrt{ }$ & $\sqrt{ }$ & Bektas and Laporte (2011) \\
\hline 9 & Time-dependent PRP & Total time and total fuel & $\sqrt{ }$ & $\sqrt{ }$ & $\sqrt{ }$ & $\sqrt{ }$ & Franceschetti et al. (2013) \\
\hline 10 & Bi-objective PRP & Fuel and travel time & $\sqrt{ }$ & $\sqrt{ }$ & $\sqrt{ }$ & $\sqrt{ }$ & Demir et al. (2014b) \\
\hline 11 & Heterogeneous PRP & $\begin{array}{l}\text { Fuel-related cost, travel } \\
\text { time-related cost, and fixed } \\
\text { vehicle cost }\end{array}$ & $\sqrt{ }$ & $\sqrt{ }$ & $\sqrt{ }$ & $\sqrt{ }$ & Koc et al. (2014) \\
\hline 12 & Pickup-delivery PRP & $\begin{array}{l}\text { Fuel-related cost and travel } \\
\text { time-related cost }\end{array}$ & $\sqrt{ }$ & $\sqrt{ }$ & & & Tajik et al. (2014) \\
\hline 13 & $\begin{array}{l}\text { Low carbon routing } \\
\text { problem }\end{array}$ & $\begin{array}{l}\text { Fuel-related cost and } \\
\text { vehicle usage cost }\end{array}$ & $\sqrt{ }$ & $\sqrt{ }$ & & & Zhang et al. (2015) \\
\hline 14 & Practical PRP & Total fuel consumption & $\sqrt{ }$ & $\sqrt{ }$ & & & Suzuki (2016) \\
\hline 15 & $\begin{array}{l}\text { Green vehicle routing and } \\
\text { scheduling problem }\end{array}$ & Total $\mathrm{CO}_{2}$ emission & $\sqrt{ }$ & $\sqrt{ }$ & & $\sqrt{ }$ & Xiao and Konak (2015) \\
\hline 16 & $\begin{array}{l}\text { Mixed-integer convex } \\
\text { programming-based PRP }\end{array}$ & $\begin{array}{l}\text { Fuel-related cost and travel } \\
\text { time-related cost }\end{array}$ & $\sqrt{ }$ & $\sqrt{ }$ & $\sqrt{ }$ & $\sqrt{ }$ & Fukasawa et al. $(2016,2017)$ \\
\hline 17 & Robust PRP & Total fuel consumption & $\sqrt{ }$ & $\sqrt{ }$ & $\sqrt{ }$ & $\sqrt{ }$ & Eshtehadi et al. (2017) \\
\hline 19 & $\begin{array}{l}\text { Variant of PRP with route } \\
\text { speed optimization }\end{array}$ & Driver's wage and fuel cost & $\sqrt{ }$ & $\sqrt{ }$ & $\sqrt{ }$ & $\sqrt{ }$ & Dabia et al. (2017) \\
\hline
\end{tabular}

\section{Linear constraints for travel time and travel speed}

The travel time $t_{i j}$ of an arc $(i, j)$ with length $D_{i j}$, calculated as $t_{i j}=D_{i j} / v_{i j}$, is a nonlinear function of the average travel speed $v_{i j}$. Owing to this known nonlinear relationship, referred to as the time-speed relation in this study, the travel speed and travel time cannot be used as continuous decision variables concurrently in a mathematical linear model. Therefore, Bektas and Laporte (2011) and Demir et al. (2012) linearized this nonlinear term through 
discretizing the speed variable $v_{i j}$ over several intervals and introducing a binary variable to determine which speed interval should be selected and the corresponding travel time to be precalculated. The speed discretization method was also used in most variants of PRP, e.g., TD-PRP by Franceschetti et al. (2013), bi-objective PRP by Demir et al. (2014b), heterogeneous PRP by Koc et al. (2014), and robust PRP by Eshtehadi et al. (2017). However, using discrete values to represent a continuous variable has its own drawbacks. First, the mathematical model becomes more difficult to solve owing to the use of additional binary variables. Second, it is difficult to estimate or control the approximation error, which may lead to sub-optimal solutions.

In this section, we introduce a linearization method for the time-speed relation with a controllable error range, which enables us to model PRP with continuous decision variables. This method can also be applied to convert many other discrete PRP models into continuous ones.

The nonlinear relationship $t_{i j}=D_{i j} / v_{i j}$ between $t_{i j}$ and $v_{i j}$ can be approximated by a set of secant line segments, denoted as $P=\left\{p_{1}, p_{2}, \ldots\right\}$, starting from the minimum speed limit $\left(v_{\min }\right)$ to the maximum speed limit $\left(v_{\max }\right)$, as shown in Fig. 1. Each secant line $p \in P$ is defined as $t_{i j}=K_{p}^{(i, j)} v_{i j}+B_{p}^{(i, j)}$ with slope $K_{p}^{(i, j)}$ and intercept $B_{p}^{(i, j)}$ passing through the points $\left(v_{p-1}, t_{p-1}\right)$ and $\left(v_{p}, t_{p}\right)$. If the problem objective involves minimizing a cost function of travel times, we can bound the travel time $t_{i j}$ on the travel speed $v_{i j}$ using the following set of linear constraints.

$$
t_{i j} \geq K_{p}^{(i, j)} v_{i j}+B_{p}^{(i, j)}, \quad \forall p \in P
$$

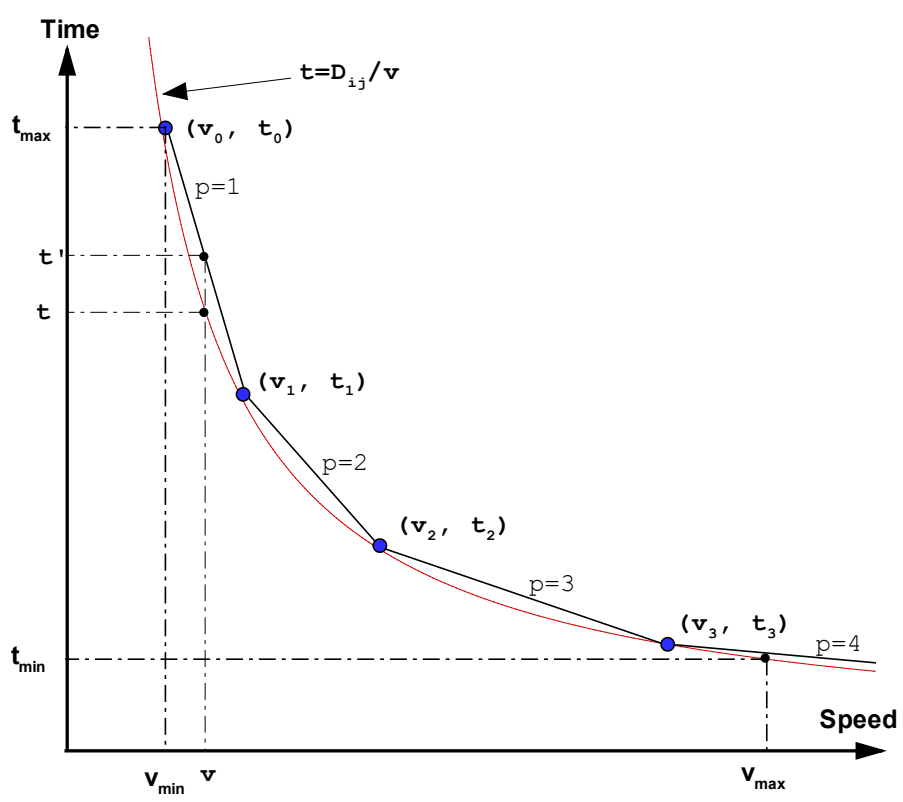

Fig. 1 Linearization of time-speed relation using secant lines

Note that the above piecewise linearization method for a nonlinear function has to be used in a condition in which the nonlinear function must be concave (or convex) when the objective function is toward the minimization (or maximization) of the function value. Similar linearization methods for expressing nonlinear relationships in a linear model can be found in Wang and Meng (2012), Sherali et al. (2003), Castillo and Westerlund (2005), Konak et al. (2006), Xiao et al. (2017), and Xie et al. (2017). 
Note that the larger the number of secant lines used, the more closely Eq. (1) can approximate the nonlinear curve $t_{i j}=D_{i j} / v_{i j}$. Let $t_{i j}^{\prime}$ be the approximated travel time, and in order for the approximation error to be less than $\varepsilon \%$, i.e., $\frac{t_{i j}^{\prime}-t_{i j}}{t_{i j}} \times 100 \leq \varepsilon \%$, the minimum number of secant lines $\eta$ can be estimated by Eq. (2) as follows (the detailed proof is provided in the Appendix):

$$
\eta=\left\lceil\frac{\ln v_{\max }-\ln v_{\min }}{\ln \left(1+2 \varepsilon+2 \sqrt{\varepsilon+\varepsilon^{2}}\right)}\right\rceil,
$$

where $v_{\min }$ and $v_{\max }$ are the minimum and maximum permissible travel speeds respectively, $\varepsilon$ is the maximum allowed percent deviation, and $\left.\Gamma^{*}\right\rceil$ denotes the smallest integer larger than or equal to *. Please note that in Eq. (2), $\eta$ depends on $v_{\min }, \quad v_{\max }$, and $\varepsilon$, but does not depend on $D_{i j}$. Therefore, the same set of secant lines can be used to linearize the curve $t_{i j}=D_{i j} / v_{i j}$ for all arcs of different lengths. In Table 2, the minimum required numbers of secant lines are provided for different $\varepsilon$ values under the speed range of $[10 \mathrm{~km} / \mathrm{h}, 120 \mathrm{~km} / \mathrm{h}]$ for an intuitive view.

Table 2 Required minimum number of secant lines for speed range [10, 120]

\begin{tabular}{c|ccccccc}
\hline Maximum allowed deviation $\varepsilon \%$ & $5.0 \%$ & $3.00 \%$ & $2.00 \%$ & $1.00 \%$ & $0.50 \%$ & $0.10 \%$ & $0.05 \%$ \\
\hline Minimum number of secant lines $n$ & 5 & 7 & 8 & 12 & 16 & 39 & 55 \\
\hline
\end{tabular}

By starting the first secant line from the minimum speed $v^{\min }$, we can deduce the following formula in Eq. (3) to determine the slope and intercept of secant line $t=K_{p}^{(i, j)} v+B_{p}^{(i, j)}, p \in P$, for a given arc $(i, j)$ with distance $D_{i j}$. The detailed derivation of Eq. (3) can be found in the Appendix section.

$$
\left\{\begin{array}{l}
K_{p}^{(i, j)}=-\frac{1}{\mu^{2 p-1} \cdot v_{\min }^{2}} \cdot D_{i j} \\
B_{p}^{(i, j)}=\frac{\mu+1}{\mu^{p} \cdot v_{\min }} \cdot D_{i j}
\end{array}, \quad \forall p=1,2, \ldots, \eta,\right.
$$

where $\mu=1+2 \varepsilon+2 \sqrt{\varepsilon+\varepsilon^{2}}, \quad v_{\min }$ is the allowed minimum travel speed on the arc $(i, j)$, and $\eta$ is the minimum number of secant lines calculated by Eq. (2). Let $K_{p}=-\frac{1}{\mu^{2 p-1} \cdot v_{\min }^{2}}$ and $B_{p}=\frac{\mu+1}{\mu^{p} \cdot v_{\min }}$, such that we have $K_{p}^{(i, j)}=K_{p} D_{i j}$ and $B_{p}^{(i, j)}=B_{p} D_{i j}$, and Eq. (1) is changed to Eq. (4) as given below:

$$
t_{i j} \geq D_{i j} K_{p} v_{i j}+D_{i j} B_{p}, \quad \forall p \in 1,2, \ldots, n .
$$

Please note the following:

(1) In Eq. (4), $K_{p}$ and $B_{p}$ are parameters that can be precalculated before solving the problem since they do not depend on distance $D_{i j}$.

(2) For the same accuracy requirement of $\varepsilon$, all arcs can use the same set of $K_{p}$ and $B_{p}$ in the linearization.

\section{Linear constraints for FCR and travel speed}

For a fossil fuel-powered vehicle, the FCR (in liters per unit distance) for traveling a given distance is a nonlinear function of the travel speed. We refer to this function hereafter as the fuel-speed relationship. In the 
literature, while there are several models that characterize the functional relationship between the fuel consumption and route variables such as travel speed, acceleration, load, and traffic conditions (see Demir et al., 2011), the most widely used models include the MEET model developed by Hickman (1999) and the CMEM model by Barth et al. (2005) and Barth and Boriboonsomsin (2008). In these two models, the fuel-speed relationship is a U-shaped convex downward function that has an optimal speed at the bottom, and higher FCRs are observed for any deviation from the optimal speed.

In this study, we develop two methods for linearizing the nonlinear fuel-speed relationship. The first is for vehicles whose FCR model conforms to the CMEM model (Section 4.1), and the second is a general numeric approximation approach for all types of fossil fuel-powered vehicles (Section 4.2).

\subsection{Linearization of fuel-speed relation for the CMEM model}

The CMEM by Barth et al. (2005) and Barth and Boriboonsomsin (2008) is an instantaneous model for estimating fuel consumption for a given distance. The FCR (in L/s), denoted as $F R$ for differentiating it from FCR, of a vehicle traveling at a constant speed $v$ (in $\mathrm{m} / \mathrm{s}$ ) with load $f($ in $\mathrm{kg}$ ) can be represented as a function of variables $v$ and $f$ as follows:

$$
F R(v, f)=\frac{\xi}{\kappa \psi}\left(k N_{e} V+\frac{0.5 C_{d} \rho A v^{3}+(\mu+f) v\left(g \sin \phi+g C_{r} \cos \phi\right)}{1000 \varepsilon \bar{\omega}}\right) .
$$

The notation and meaning of the parameters in Eq. (5) are presented in Table A1 in the Appendix. The FCR for traveling a unit of distance $(1 \mathrm{~m})$, which takes a time of $1 / v \mathrm{~s}$, denoted as $F($ in $\mathrm{L} / \mathrm{m})$, can be written as

$$
\begin{aligned}
F(v, f) & =F R / v \\
& =\frac{\xi k N_{e} V}{\kappa \psi} v^{-1}+\frac{0.5 C_{d} A \rho \xi}{1000 \kappa \psi \varepsilon \bar{\omega}} v^{2}+\frac{\mu \xi\left(g \sin \phi+g C_{r} \cos \phi\right)}{1000 \kappa \psi \varepsilon \bar{\omega}}+\frac{f \xi\left(g \sin \phi+g C_{r} \cos \phi\right)}{1000 \kappa \psi \varepsilon \bar{\omega}} f .
\end{aligned}
$$

By using parameters $\alpha=\xi k N_{e} V /(\kappa \psi), \quad \beta=0.5 C_{d} A \rho \xi /(1000 \kappa \psi \varepsilon \bar{\omega}), \quad \gamma=\mu \xi\left(g \sin \phi+g C_{r} \cos \phi\right) /(1000 \kappa \psi \varepsilon \bar{\omega})$, and $\varphi=f \xi\left(g \sin \phi+g C_{r} \cos \phi\right) /(1000 \kappa \psi \varepsilon \bar{\omega})$ in the equation above, the fuel consumption function can be simplified as

$$
F(v, f)=\alpha v^{-1}+\beta v^{2}+\gamma+\varphi f .
$$

Let $F_{1}(v)=\alpha v^{-1}, F_{2}(v)=\beta v^{2}, F^{\prime}(v)=F_{1}(v)+F_{2}(v)+\gamma$, and $F^{*}(f)=\varphi f$, and the above Eq. (7) can be represented as

$$
F(v, f)=F^{\prime}(v)+F^{*}(f)=F_{1}(v)+F_{2}(v)+\gamma+\varphi f,
$$

where $F^{\prime}(v)$ represents the amount of fuel consumed by an unloaded vehicle when traveling one unit of distance, and $F^{*}(f)$ represents the additional fuel consumed owing to the vehicle's payload.

Because the first term of Eq. (7), i.e., $F_{1}(v)=\alpha v^{-1}$, has the same mathematical expression as the time-speed relationship discussed in Section 3, we can directly use a similar set of linear constraints as in Eq. (4) to linearize it as follows:

$$
F_{1} \geq \alpha K_{p} v+\alpha B_{p}, \quad \forall p \in 1,2, \ldots, \eta
$$

where $K_{p}$ and $B_{p}$ are also calculated as $K_{p}=-\frac{1}{\mu^{2 p-1} \cdot v_{\min }^{2}}$ and $B_{p}=\frac{\mu+1}{\mu^{p} \cdot v_{\min }} ; \mu=1+2 \varepsilon+2 \sqrt{\varepsilon+\varepsilon^{2}}$, and $\varepsilon$ is the maximum allowed deviation from the actual value. 
Because the third and fourth parts of Eq. (7), i.e., $\gamma+\varphi f$, are already linear, we just focus on the linearization of the second term, i.e., $F_{2}=\beta v^{2}$. First, we remove the coefficient $\beta$ from the term $F_{2}=\beta v^{2}$ and use a set of secant lines to surrogate the basic nonlinear curve $y=x^{2}$, as shown in Fig. 2.

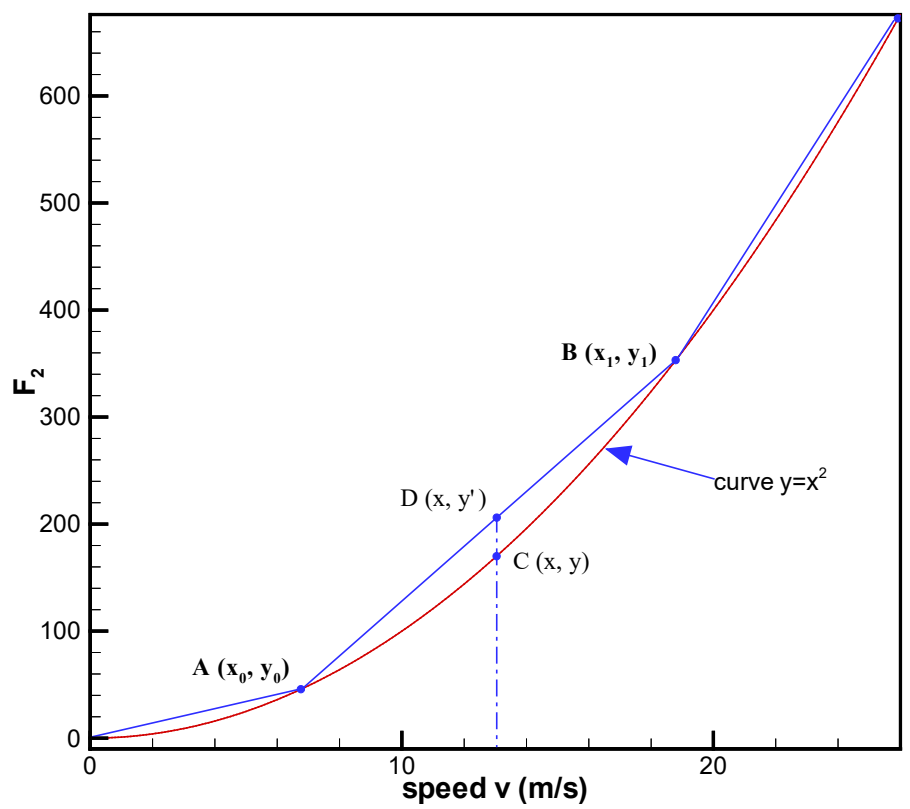

Fig. 2 Linearization of nonlinear curve $y=x^{2}$ using secant lines

For two points $\mathrm{A}\left(x_{0}, y_{0}\right)$ and $\mathrm{B}\left(x_{1}, y_{1}\right)$, where $x_{0}>0$ and $x_{1}>0$, on the curve $y=x^{2}$, as shown in Fig. 2, the equation of secant line A-B can be written as $y=\left(x_{1}+x_{0}\right) x-x_{0} x_{1}$. For any $x$ value such that $x_{0}<x<x_{1}$; let $\mathrm{D}\left(x, y^{\prime}\right)$ represent the approximated point to vertically connect the actual point $\mathrm{C}(x, y)$ on the curve as shown in Fig. 2. In our approach, $y^{\prime}$ is used as a surrogate value for $y$. Then, the relative error in this approximation at $x$ is defined as $d(x)=\frac{y^{\prime}-y}{y}=\left(x_{1}+x_{0}\right) x^{-1}-x_{0} x_{1} x^{-2}-1$. Given $x_{0}$, the next point $x_{1}$ that ensures $d(x) \leq \varepsilon$ at any $x$ value that maximizes the relative error between $x_{0}$ and $x_{1}$ can be found by taking the derivative of $d(x)$. By setting the derivative $d^{\prime}(x)=0$ under constraint $d(x) \leq \varepsilon$, we can find the recursive formula in Eq. (10) to determine the farthest point $\mathrm{B}\left(x_{p}, y_{p}\right)$ on curve $y=x^{2}$ starting from point $\mathrm{A}\left(x_{p-1}, y_{p-1}\right)$ for $p=1,2,3 \ldots$ Thus, the maximum relative error of using line segment A-B as a surrogate of curve segment A-B is less than $\varepsilon$.

$$
x_{p}=\mu x_{p-1}, y_{p}=x_{p}^{2} \quad p=1,2,3, \ldots,
$$

where $\mu=1+2 \varepsilon \pm 2 \sqrt{\varepsilon(1+\varepsilon)}$. Therefore, we can use a set of secant lines to approximate the curve segment of $F_{2}=v^{2}$ from the minimum travel speed $v_{\min }$ to the maximum travel speed $v_{\max }$. The slopes and intercepts of the secant lines are determined by the following equation (see Appendix for the detailed proof and deduction).

$$
\left\{\begin{array}{l}
k_{p}=(\mu+1) \mu^{p-1} v_{\min } \\
b_{p}=-\mu^{2 p-1} v_{\min }^{2}
\end{array} \quad p=1,2, \ldots \eta,\right.
$$

where $k_{p}$ and $b_{p}$ are the slope and intercept of the $p^{\text {th }}$ line respectively, and $\eta$ is the minimum number of lines that guarantees the maximum deviation within a given value of parameter $\varepsilon$. Note that $\eta$ is also calculated using Eq. (2) based on parameter $\varepsilon$. 
Thus, the nonlinear expression $F_{2}(v)=\beta v^{2}$ can be approximated by a set of linear constraints to guarantee the maximum deviation within a given value of parameter $\varepsilon$ as follows:

$$
F_{2} \geq \beta k_{p} v+\beta b_{p}, \quad \forall p \in 1,2, \ldots, \eta .
$$

Note that $k_{p}$ and $b_{p}$ in Eq. (12), and $K_{p}$ and $B_{p}$ in Eq. (4) and Eq. (9), regardless of the vehicle type, are all determined only by $\varepsilon, v_{\min }$, and $v_{\max }$, as long as the CMEM model applies to the vehicle's FCR.

\subsection{General numeric approach for fuel-speed relationship linearization}

In this section, we provide a general linearization approach based on a numeric calculation of the fuel-speed relationship of other types of fuel consumption (or $\mathrm{CO}_{2}$ emissions) models, such as the MEET model based on a regression equation developed by Hickman (1999) or any empirical fuel-speed functions provided by vehicle manufacturers. The fuel-speed relationship of a fossil fuel-powered vehicle is typically expressed as a U-shaped curve with the most economical speed point at the bottom as shown in Fig. 3. In this figure, the emission-speed curve of a 7.5 to 16-ton diesel truck is plotted using the MEET model of Hickman (1999), which is a regression function in the form of $E R(v)=K+A v+B v^{2}+C v^{3}+\frac{D}{v}+\frac{E}{v^{2}}+\frac{F}{v^{3}}$, where $K=871, A=-16, B=0.143, C=0, D$ $=0, E=32031$, and $F=0$. Note that because the volume of $\mathrm{CO}_{2}$ emissions is proportional to the amount of fuel consumed, the vehicle's fuel-speed curve has also a similar shape to the emission-speed curve.

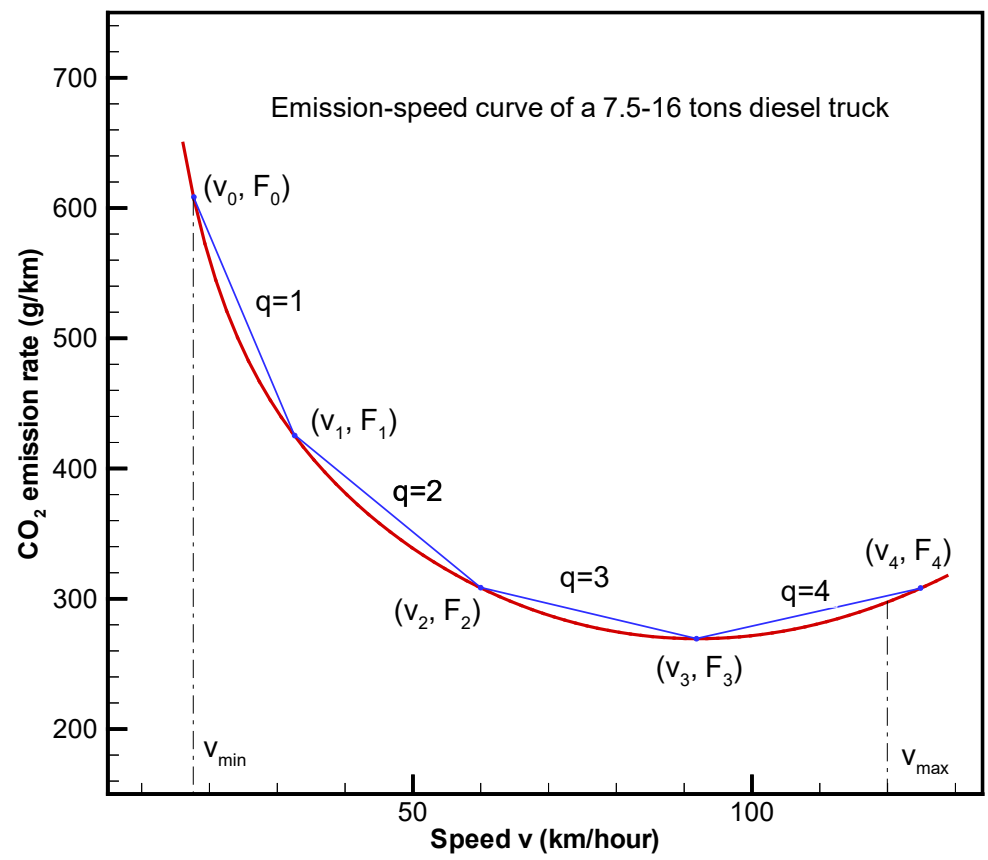

Fig. 3 General linearization approach using secant lines

The proposed general approach uses a set of secant lines to approximate the $f u e l$-speed function $F(v)$. Suppose that these lines are formed by points $\left(v_{0}, F_{0}\right),\left(v_{1}, F_{1}\right),\left(v_{2}, F_{2}\right), \ldots,\left(v_{\eta}, F_{\eta}\right)$ on the curve of $F(v)$ as shown in Fig. 3, where $v_{0}=v_{\min }, v_{\eta} \geq v_{\max }$, and $\eta$ is the minimum number of secant lines for guaranteeing that the maximum deviation is within the accuracy limit $\varepsilon$. For two points on the fuel-speed curve $F(v)$, e.g., $A\left(v_{0}, F_{0}\right)$ and $B\left(v_{1}, F_{1}\right)$, the secant line going through points $A$ and $B$ is expressed as $F^{\prime}(v)=k^{\prime} v+b^{\prime}$. The percent deviation of $F^{\prime}(v)$ from $F(v)$ can be expressed as $D(v)=100 \% \times\left[F^{\prime}(v)-F(v)\right] / F(v)$. For the first line, we set $v_{0} \leftarrow v_{\min }$ and 
$v_{1} \leftarrow v_{\min }$ and increase $v_{1}$ gradually by a small number $\Delta$ (i.e., $v_{1} \leftarrow v_{1}+\Delta$ with $\Delta=1 \mathrm{~km} / \mathrm{h}$ ). Then, we check all discretized points $v \in\left[v_{0}, v_{1}\right]$, starting from $v_{0}$ (by $v \leftarrow v_{0}$ ) increased gradually by a smaller step $\delta$ (by $v \leftarrow v+\delta$ ), which is much smaller than $\Delta$, e.g., $\delta=0.1 \mathrm{~km} / \mathrm{h}$. During this process, if $D(v) \leq \varepsilon$ is satisfied, then we continue to increase $v_{1}$ by $v_{1} \leftarrow v_{1}+\Delta$; otherwise, we set $v_{1}$ as the end of the first line and continue to determine $v_{2}$ starting from $v_{1}$ for the second line until the last line is determined. This process is described by the Line gen $\left(v_{\min }, v_{\max }, \Delta\right.$, $\delta, \varepsilon)$ procedure in Fig. 4.

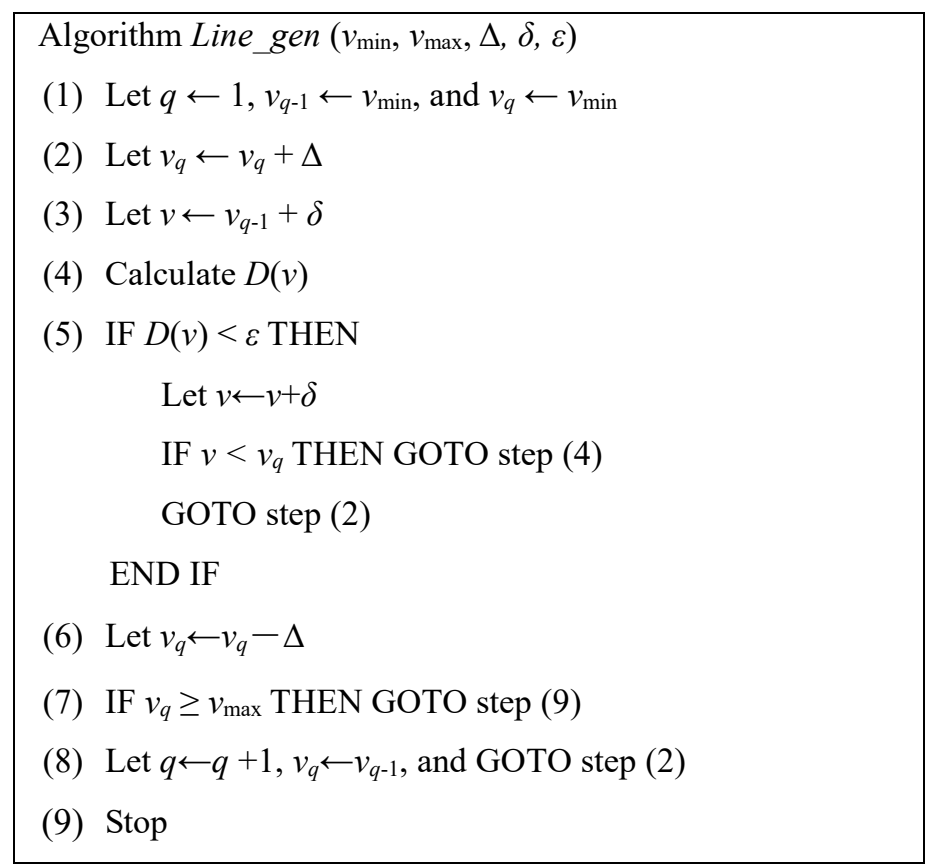

Fig. 4 Numeric calculation procedure to generate secant lines

Using the procedure in Fig. 4 , the minimum number of discrete points, i.e., $\left(v_{0}, F_{0}\right),\left(v_{1}, F_{1}\right),\left(v_{2}, F_{2}\right), \ldots,\left(v_{\eta}, F_{\eta}\right)$, on the fuel-speed curve can be obtained, and each pair of two consecutive points, i.e., $\left(v_{q-1}, F_{q-1}\right)$ and $\left(v_{q}, F_{q}\right)$, form a secant line $F^{\prime}=k_{q}^{\prime} v+b_{q}^{\prime}$, where $q=1,2, \ldots, \eta$. For an $\operatorname{arc}(i, j)$ with a distance $D_{i j}$ (in $\left.\mathrm{m}\right)$ traveled by an empty vehicle with the speed of $v_{i j}$, the fuel consumption $F^{\prime}{ }_{i j}$ can be bounded by linear constraints as follows:

$$
F_{i j}^{\prime} \geq D_{i j} k_{q}^{\prime} v_{i j}+D_{i j} b_{q}^{\prime} \quad \forall q=1,2, \ldots, \eta
$$

Note that because $k_{q}^{\prime}$ and $b_{q}^{\prime}$ in Eq. (13) depend on the vehicle type, multiple sets of $k_{q}^{\prime}$ and $b_{q}^{\prime}$ need to be precalculated for each vehicle type in a heterogeneous PRP with different vehicle types. The numerical approach for the fuel-speed linearization has the advantage of computational efficiency because it linearizes the fuel-speed curve as a whole whereas the CMEM model needs to linearize two separate curves.

\section{Continuous pollution routing problem}

In this section, we extend the discrete PRP of Bektas and Laporte (2011) to a general case, i.e., the CPRP, in which all scheduling variables including travel speeds, travel time, FCR, payloads, and departure/arrival/waiting times are treated as continuous decision variables simultaneously. The CPRP is described on a directed graph $G=(N, A)$, where $N=\{0,1,2, \ldots, n\}$ is the set of nodes representing the customers and depot (denoted by 0$)$ and $A=\{(i, j) \mid i, j \in N ; i \neq j\}$ is the set of feasible arcs that connect the customers/depot. A fleet of homogenous 
vehicles is dispatched to serve the customers starting from and returning to the depot. Each $\operatorname{arc}(i, j)$ in $A$ has a known distance. Moreover, each customer in $N$ has a known demand, a duration of service time, and a time window in which the service must start. Furthermore, each vehicle/driver has a unit cost per working time, a load capacity limit, and an identified fuel consumption model. The aim is to determine a set of optimal routes for vehicles and simultaneously optimize the travel schedules, including the travel speeds, travel time, and payloads over each arc and the departure time, arrival time, and waiting time at each node, in order to minimize a total cost function that includes two components: (1) the operational costs proportionally related to the total delivery time and (2) the environmental cost proportionally related to the total fuel consumption. In the following subsections, we first present an $\varepsilon$-accurate mixed-integer linear programming (MILP) model for the CPRP, and then provide a post-optimization procedure for eliminating the approximation errors and some propositions regarding the feasibility and optimality of a solution found by the model.

\subsection{The $\varepsilon$-accurate mixed-integer linear programming model of CPRP}

In the following, we present an $\varepsilon$-CPRP based on the linearization approaches introduced in Sections 3 and 4. The parameters and decision variables used in $\varepsilon$-CPRP are listed as follows ${ }^{1}$.

Parameter notations:

$\varepsilon \quad$ maximum allowable percent error in the linearization of time, speed, and fuel consumption relationships

$i, j \quad$ index of nodes, $i=0,1,2, \ldots, n$ (the depot is represented by node 0 )

$n \quad$ total number of nodes (including the depot)

$N \quad$ set of nodes, including the depot

$N^{\prime} \quad$ set of customer nodes, $N^{\prime}=N \backslash\{0\}$

$A \quad$ set of arcs formed by all pairs of nodes, $A=\{(i, j): \forall i \in N, j \in N, i \neq j\}$

$A^{\prime} \quad$ set of arcs formed by all pairs of customer nodes, $A^{\prime}=\left\{(i, j): \forall i \in N^{\prime}, j \in N^{\prime}, i \neq j\right\}$

$D_{i j} \quad$ distance of $\operatorname{arc}(i, j)$

$\bar{v}_{i j}, \underline{v}_{i j} \quad$ upper and lower travel speed limits on arc $(i, j)$, respectively

$d_{i} \quad$ demand requirement of customer $i$

$g_{i} \quad$ service time of customer $i$

$\left[S_{i}, E_{i}\right]$ time window for serving customer $i$; $\left[S_{0}, E_{0}\right]$ represent the entire planning horizon, and $S_{0}=0$

C maximum payload of vehicle

$o \quad$ number of available homogeneous vehicles in the fleet

$r \quad$ binary parameter indicating if the vehicle conforms to the CMEM model $(r=1)$ or not $(r=0)$

$P \quad$ set of secant lines predetermined based on parameter $\varepsilon$ for the time-speed linearization

$K_{p}, B_{p} \quad$ slope and intercept respectively of the $p^{\text {th }}$ secant line in $P, \quad p \in P$

$Q \quad$ set of predetermined secant lines based on parameter $\varepsilon$ for the linearization of the second term of the CMEM fuel-speed model

$k_{p}, b_{p} \quad$ slope and intercept respectively of the $p^{\text {th }}$ secant line in $Q, \quad p \in Q$

\footnotetext{
${ }^{1}$ Note that notations defined in Table A2 are only for the CMEM model in Section 4.1, some of which are redefined in the $\varepsilon$-CPRP model.
} 
$\alpha, \beta, \gamma \quad$ coefficients of the CMEM fuel-speed model in Eq. (7)

$\varphi \quad$ unit FCR $(\mathrm{L} / \mathrm{m})$ of the vehicle traveling with one additional unit of load $(\mathrm{kg})$

$Q^{\prime} \quad$ set of predetermined secant lines for the general numeric approach to the fuel-speed linearization

$k_{p}^{\prime}, b_{p}^{\prime} \quad$ slope and intercept of the $p^{\text {th }}$ secant line in $Q^{\prime}, \quad p \in Q^{\prime}$

$f_{c} \quad$ fuel cost per liter of fuel $(£)$

$d_{c} \quad$ driver wage $(£ / \mathrm{s})$

$\bar{F} \quad$ a large number greater than the maximum possible value of FCR

Binary decision variables:

$X_{i j} \quad X_{i j}=1$ if $\operatorname{arc}(i, j)$ is traveled by a vehicle; $X_{i j}=0$ otherwise

Non-negative continuous decision variables:

$v_{i j} \quad$ travel speed $(\mathrm{m} / \mathrm{s})$ on $\operatorname{arc}(i, j)$

$t_{i j} \quad$ travel time (s) spent on $\operatorname{arc}(i, j)$

$F_{i j} \quad \mathrm{FCR}(\mathrm{L} / \mathrm{m})$ of the vehicle traveling on $\operatorname{arc}(i, j)$ with a payload

$F^{\prime}{ }_{i j} \quad$ no-load FCR $(\mathrm{L} / \mathrm{m})$ of the vehicle traveling on arc $(i, j)$ with zero payload

$F_{1 i j}, F_{2 i j} \quad$ the first and second terms respectively of the CMEM fuel-speed model in Eq. (8)

$a_{i} \quad$ arriving time (s) at customer $i$

$w_{i} \quad$ vehicle waiting time (s) at node $i$

$s_{i} \quad$ time (s) of departing from depot to node $i$ if node $i$ is the first node visited by vehicle; 0 otherwise

$f_{i j} \quad$ load $(\mathrm{kg})$ of vehicle on $\operatorname{arc}(i, j)$

The objective function used in $\varepsilon$-CPRP is consistent with the comprehensive cost function used by Bektas and Laporte (2011), Demir et al. (2012), Kramer et al. (2015b), Franceschetti et al. (2013), and Eshtehadi et al. (2017), which contains two components: (1) the driver's wage proportionally related to the total delivery time, and (2) the fuel cost proportionally related to the total amount of fuel used. In addition, we adopt the two policies discussed by Franceschetti et al. (2013) for the driver payment. In the first policy, drivers are paid for the period from the beginning of the planning horizon until they return to the depot (DPFB for short). In the second policy, drivers are paid for the duration from their departure times to their return times to the depot (DPFD for short). The difference is that the time waiting at the depot is not paid under policy DPFD but is paid under policy DPFB. The objective functions under the two policies are formulated as Eq. (14a) and Eq. (14b), respectively. Note that variable $s_{i}$ is introduced to calculate the waiting time at the depot for each route before the departure.

Thus, the $\varepsilon$-accurate MILP model for the CPRP can be formulated as follows:

$$
\begin{aligned}
& \text { Min. Total Cost }(D P F B)=\sum_{(i, j) \in A}\left(F_{i j} D_{i j} f_{c}+t_{i j} d_{c}\right)+\sum_{i \in N^{\prime}}\left(s_{i}+g_{i}+w_{i}\right) d_{c} \\
& \text { Min. Total Cost }(D P F D)=\sum_{(i, j) \in A}\left(F_{i j} D_{i j} f_{c}+t_{i j} d_{c}\right)+\sum_{i \in N^{\prime}}\left(g_{i}+w_{i}\right) d_{c}
\end{aligned}
$$

Subject to

$$
\text { (1) } \sum_{(i, j) \in A} X_{i j}=1 \quad \forall j \in N^{\prime}
$$



(2) $\sum_{(i, j) \in A} X_{i j}=1$
$\forall i \in N^{\prime}$
(3) $\sum_{j \in N^{\prime}} X_{0 j} \leq o$
$\forall i \in N^{\prime}$
(4-1) $a_{j}-a_{i} \geq t_{i j}+g_{i}+w_{i}-E_{0}\left(1-X_{i j}\right)$
$\forall(i, j) \in A^{\prime}$
(4-2) $a_{j}-a_{i} \leq t_{i j}+g_{i}+w_{i}+E_{0}\left(1-X_{i j}\right)$
$\forall(i, j) \in A^{\prime}$
(4-3) $a_{j} \geq t_{0 j}-E_{0}\left(1-X_{0 j}\right)$
$\forall j \in N^{\prime}$
(5) $t_{i j} \geq D_{i j} K_{p} v_{i j}+D_{i j} B_{p}-E_{0}\left(1-X_{i j}\right)$
$\forall(i, j) \in A, p \in P$
(6-1) $F_{1 i j} \geq \alpha K_{p} v_{i j}+\alpha B_{p}-\bar{F}\left(1-X_{i j}\right)$
$\forall(i, j) \in A, p \in P, r=1$
(6-2) $F_{2 i j} \geq \beta k_{p} v_{i j}+\beta b_{p}-\bar{F}\left(1-X_{i j}\right)$
$\forall(i, j) \in A, p \in Q, r=1$
(6-3) $F_{i j}^{\prime} \geq F_{1 i j}+F_{2 i j}+\gamma X_{i j}$
$\forall(i, j) \in A, r=1$
(6-4) $\quad F_{i j}^{\prime} \geq k_{p}^{\prime} v_{i j}+b_{p}^{\prime}-\bar{F}\left(1-X_{i j}\right)$
$\forall(i, j) \in A, p \in Q^{\prime}, r=0$
(7) $F_{i j}=F_{i j}^{\prime}+\varphi f_{i j}$
$\forall(i, j) \in A$
(8) $\quad \underline{v}_{i j} X_{i j} \leq v_{i j} \leq \bar{v}_{i j} X_{i j}$
$\forall(i, j) \in A$
(9) $S_{i} \leq a_{i} \leq E_{i}$
$\forall i \in N^{\prime}$
(10) $\sum_{(j, i) \in A} f_{j i}-\sum_{(i, j) \in A} f_{i j}=d_{i}$
$\forall i \in N^{\prime}$
(11) $f_{i j} \leq C X_{i j}$
$\forall(i, j) \in A$
(12) $s_{i} \geq a_{i}-t_{0 i}-E_{0}\left(1-X_{0 i}\right)$
$\forall i \in N^{\prime}$
(13) $\left\{\begin{array}{l}X_{i j} \in\{0,1\} ; v_{i j} \geq 0 ; t_{i j} \geq 0 ; a_{i} \geq 0 ; w_{i} \geq 0 ; s_{i} \geq 0 \\ F_{i j} \geq 0 ; F_{0 i j} \geq 0 ; F_{1 i j} \geq 0 ; F_{2 i j} \geq 0 ; f_{i j} \geq 0\end{array}\right.$
$\forall i \in N^{\prime},(i, j) \in A$

In the above model, Constraints (1) and (2) ensure that each customer is visited only once. Constraint (3) restricts the number of vehicles used in a solution to the given upper limit. Constraints (4-1) and (4-2) are a set of disjunctive constraints to calculate arrival time $a_{j}$ at node $j$ after traveling arc $(i, j)$. Note that Constraints (4-1) and (4-2) are not applicable to the arcs starting from or terminating at the depot. Constraint (4-3) requires that the arrival time to the first customer of a vehicle must be greater than or equal to the travel time spent on the first arc. Note that jointly, Constraints (4-1)-(4-3) calculates exact values for vehicles' arriving time at each node. Constraint (5) is the linearization of the time-peed relationship using a set of secant lines to approximate the nonlinear curve of $t_{i j}=D_{i j} / v_{i j}$ as discussed in Section 3. Similarly, Constraints (6-1) and (6-2) use a set of secant lines to linearize the first and second terms of the CMEM fuel-speed model in Eq. (8), respectively (see Section 4.1 for details). Constraints (6-3) and (6-4) calculate the no-load FCR (L/m) of a vehicle traveling on arc $(i, j)$ for cases whether the vehicle conforms to the CMEM emission model $(r=1)$ or not $(r=0)$, respectively. Note that only one of Constraints (6-3) and (6-4) will be active depending on parameter $r$. Constraint (7) calculates the FCR $(\mathrm{L} / \mathrm{m})$ of the vehicle with payload $f_{i j}$ on $\operatorname{arc}(i, j)$. Constraint (8) restricts the travel speed within the speed limit range. Constraint 
(9) ensures that the service starting times of all customers are within their time windows. Constraint (10) computes the vehicle's payload along the tour. Constraint (11) limits the vehicle's payload by its maximum payload capacity $C$ and forces $f_{i j}$ to be zero if arc $(i, j)$ is not selected in a tour. Constraint (12) bounds the waiting time at the depot and sets the departing time from the depot for the first visited nodes. Note that $s_{i}$ will be forced to be zero if node $i$ is not a first visited node. Constraint (13) defines domains of decision variables.

Constraints (4), (5), (6), and (12) are only enforced when $X_{i j}=1$. The possible subtours in a solution are eliminated by either of Constraint (4) or Constraint (10). Constraint (6-1), (6-2), and (6-3) are only used when $r=1$, whereas Constraint (6-4) is used when $r=0$.

The $\varepsilon$-CPRP model contains two types of linearization method for the FCR introduced in Section 4. However, only one of them will be applied to solve a problem instance as specified by parameter $r$. For simplicity, we use $\varepsilon$-CPRP to refer to the case in which $r=1$, indicating that the CMEM linearization method is applied, and $\varepsilon$-CPRP to refer to the case in which $r=0$, indicating that the general numeric approximation approach is applied. Computational experiments on $\varepsilon-C P R P$ and $\varepsilon-C P R P^{\prime}$ models presented in the next section show that both models have the same level of accuracy but the $\varepsilon-C P R P^{\prime}$ model has a higher computational efficiency (using approximately $50 \%$ less CPU time) than the $\varepsilon$-CPRP model on average. This result is mainly due to one additional set of secant lines used in the $\varepsilon$-CPRP model for the linearization of the fuel-speed relationship.

\subsection{Post-optimization procedure and theoretical analysis}

Owing to the linearization approximation of the time-speed relation, a feasible solution of $\varepsilon$-CPRP is only an approximate solution to the CPRP, which has two approximation errors: (1) the travel time difference between the approximated travel time by Constraint (5) and the actual time calculated by $t_{i j}=D_{i j} / v_{i j}$, and (2) the FCR difference between the approximated no-load FCR by Constraints (6-1) to (6-4) and the actual no-load FCR calculated by $F_{i j}^{\prime}=\alpha v_{i j}^{-1}+\beta v_{i j}^{2}+\gamma$. We apply a post-optimization procedure on a feasible solution, noted as $\pi$, found by the $\varepsilon$-CPRP model to eliminate approximation errors. The post-optimization procedure first fixes all obtained values of decision variables $x_{i j}$ and $v_{i j}$ in solution $\pi$ and then recalculates the corresponding travel times and the no-load FCRs

of all arcs by using $t_{i j}=D_{i j} / v_{i j}$ and $F_{i j}^{\prime}=\alpha v_{i j}^{-1}+\beta v_{i j}^{2}+\gamma$, respectively. After that, all values of variables $x_{i j}, v_{i j}, t_{i j}, F_{i j}^{\prime}$, and $f_{i j}$ are treated as input parameters so that a simplified MILP model including only Eq. (14) and Constraints (4), (7), (9), and (12) can be solved to obtain an accurate solution $\left(\pi^{*}\right)$. Note that solving the simplified MILP model is very efficient because the route and speed decision variables have already been fixed, and the MIP solver only needs to re-optimize the decision variables related to departure, arrival, and waiting times to align with the time window constraints again to generate a feasible solution. The main steps of the post-optimization procedure are outlined in Fig. 5. 


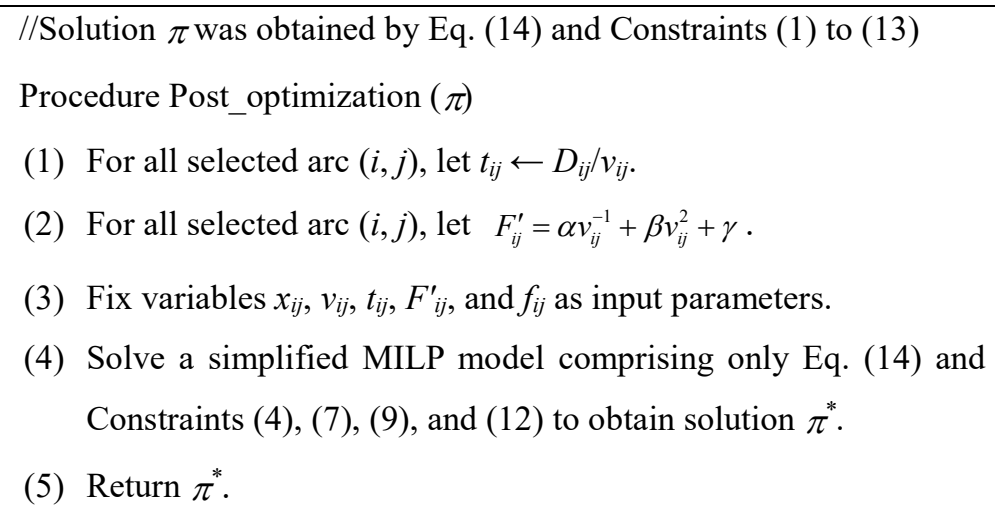

Fig. 5 Main steps of the post_optimization procedure

Theorem 1. FEASIBILITY: For a feasible solution $\pi$ of the $\varepsilon$-CPRP formulated by Eq. (14) and Constraints (1) to (13), there always exists a feasible solution $\pi^{*}$ of the original CPRP that has the same routes and travel speeds with $\pi$ and has a lower (or equal) objective value.

Proof. The proof for the feasibility of solution $\pi^{*}$ is based on the fact that the linearization methods described in this study are polyhedral inner approximations that always overestimate. Suppose $\pi$ is a feasible solution of $\varepsilon$-CPRP and $c$ represents the objective value of $\pi$. Let $t_{i j}$ be the travel time, and $F_{i j}^{\prime}$ be the no-load FCR on arc $(i, j)$ in solution $\pi$. According to the secant line-based linearization methods used in $\varepsilon$-CPRP, we will have $t_{i j} \geq t^{*}{ }_{i j}$, where $t^{*}{ }_{i j}$ is the actual travel time of $\operatorname{arc}(i, j)$ calculated by $t_{i j}^{*}=D_{i j} / v_{i j}$, and ${F^{\prime}}_{i j} \geq{F^{\prime}}^{*}{ }_{i j}$, where ${F^{\prime}}^{*}{ }_{i j}$ is the accurate no-load FCR on arc $(i, j)$ calculated by $F_{i j}^{\prime}=\alpha v_{i j}^{-1}+\beta v_{i j}^{2}+\gamma$. We can always transform solution $\pi$ to $\pi^{*}$ by using the following three steps: (1) replace $t_{i j}$ with $t^{*}{ }_{i j}$ for all arcs on the routes of solution $\pi$, (2) replace $F_{i j}^{\prime}$ with ${F^{\prime}}^{*}{ }_{i j}$ for all arcs on the routes of solution $\pi$, and (3) add $t_{i j}-t^{*}{ }_{i j}$ into the waiting time at customer/depot nodes to keep the arrival time of all customers unchanged. Thus, the new solution $\pi^{*}$ satisfies all requirements of the original CPRP, and therefore, it is a feasible solution of CPRP. Furthermore, because the total time in $\pi^{*}$ is the same as that in $\pi$, the new no-load FCR $F^{\prime *}{ }_{i j}$ is equal to or smaller than $F_{i j}^{\prime}$, and the FCR remains unchanged as there is no change in payload; thus, the objective value of solution $\pi^{*}$ is smaller than or equal to that of $\pi$.

According to Theorem 1, we can always use the post-optimization procedure in Fig. 5 to convert any feasible solution of the $\varepsilon$-CPRP model into a feasible solution of the original CPRP. Thus, we have the following Proposition 1.

Proposition 1. A feasible solution of $\varepsilon$-CPRP, after being optimized by the post-optimization procedure given in Fig. 5, is a feasible solution of the original CPRP.

Furthermore, because Constraints (5) and (6) in $\varepsilon$-CPRP join to exert tighter restrictions on variables $t_{i j}$ and $F_{i j}^{\prime}$ against the minimization of the objective value, the optimal solution obtained by $\varepsilon$-CPRP should always be worse than or equal to the optimal solution of CPRP. Thus, we come to the following Proposition 2.

Proposition 2. Let $\pi$ be an optimal solution obtained by $\varepsilon$-CPRP and $\pi^{*}$ be the optimal solution of the original CPRP; the objective value of $\pi$ is always greater than or equal to that of $\pi^{*}$.

Theorem 2. OPTIMALITY: For an optimal solution $\pi^{*}$ of CPRP, there always exists a feasible solution $\pi^{\prime \prime}$ of 
$\varepsilon$-CPRP such that $\pi^{*}$ and $\pi^{\prime \prime}$ have the same routes and travel times on each arc of routes under the condition that the upper speed limit $\bar{v}_{i j}$ is increased to $\bar{v}_{i j}(1+\varepsilon)$ for each arc $(i, j)$ in $\varepsilon$-CPRP.

Proof. This proof includes two parts as follows.

Part 1: Assume that $\pi^{*}$ is an optimal solution of CPRP. Solution $\pi^{*}$ will satisfy Constraints (1)-(4) and Constraints (7)-(13) of $\varepsilon$-CPRP because these constraints are also required by CPRP but may not satisfy Constraints (5) and (6). We use the following two steps to transform solution $\pi^{*}$ to a new solution $\pi^{\prime \prime}$ that satisfies all constraints including Constraints (5) and (6). First, by multiplying the travel speeds of all arcs in $\pi^{*}$ by $1+\varepsilon$, we transform $\pi^{*}$ to $\pi^{\prime}$ such that Constraint (5) will be satisfied (see Part 2 for the proof of this). Second, because Constraint (6) is designed to guarantee the no-load FCRs to be no less than their accurate values (calculated by $F_{i j}^{\prime}=\alpha v_{i j}^{-1}+\beta v_{i j}^{2}+\gamma$ ), we can always replace $F_{i j}^{\prime}$ of $\pi^{\prime}$ with a larger value (which transforms again $\pi^{\prime}$ to $\pi^{\prime \prime}$ ) to satisfy Constraint (6). Thus, we obtain a new feasible solution $\pi^{\prime \prime}$ that satisfies all constraints of $\varepsilon$-CPRP, including Constraints (5) and (6), and has the same routes and travel time as $\pi^{*}$. Because the travel speeds are multiplied by $1+\varepsilon$ in the first step, the upper speed limits of all arcs also need to be increased to $\bar{v}_{i j}(1+\varepsilon)$ in order to maintain the solution feasibility.

Part 2: This part of proof shows that a pair of $v_{i j}$ and $t_{i j}^{*}$ satisfies Constraint (5) if $v_{i j}=(1+\varepsilon) v_{i j}^{*}$ and $t_{i j}^{*}=D_{i j} / v_{i j}^{*}$. Constraint (5) uses a set of secant lines of the nonlinear curve $t_{i j}^{*}=D_{i j} / v_{i j}^{*}$ to guarantee that $t_{i j} \geq t_{i j}^{*}=D_{i j} / v_{i j}^{*}$ and the minimum $t_{i j}$ satisfying Constraint (5) satisfies $\frac{t_{i j}-t_{i j}^{*}}{t_{i j}^{*}} \leq \varepsilon$ for a given travel speed $v_{i j}^{*}$. This leads to $t_{i j} \leq(1+\varepsilon) t_{i j}^{*}=(1+\varepsilon) D_{i j} / v_{i j}^{*}$, which indicates that any pair of $\left(v_{i j}^{*}, t_{i j}\right)$ will satisfy Constraint (5) if $t_{i j} \geq(1+\varepsilon) D_{i j} / v_{i j}^{*}$ holds. This condition can be rewritten as $v_{i j}^{*} \geq(1+\varepsilon) D_{i j} / t_{i j}$. Thus, a pair of $v_{i j}$ and $t_{i j}^{*}$ will satisfy Constraint (5) if $v_{i j} \geq(1+\varepsilon) D_{i j} / t_{i j}^{*}=(1+\varepsilon) v_{i j}^{*}$ holds.

Note that in Theorem 2, solution $\pi^{\prime \prime}$ of $\varepsilon$-CPRP has the same routes and travel times as $\pi^{*}$. Therefore, the cost related to travel times is the same; however, the fuel cost may be increased owing to (1) the increase in travel speed by $\varepsilon \%$ and (2) FCR-speed linearization. In the FCR function $F_{i j}\left(v_{i j}\right)=\alpha v_{i j}^{-1}+\beta v_{i j}^{2}+\gamma+\varphi f_{i j}$, only the first two terms, i.e., $F_{i j}^{\prime \prime}\left(v_{i j}\right)=\alpha v_{i j}^{-1}+\beta v_{i j}^{2}$ will be affected by the increase in travel speed, which can be represented by $F_{i j}^{\prime \prime}\left(v_{i j}+\varepsilon v_{i j}\right)$. The $F C R$-speed linearization may also increase $F_{i j}^{\prime \prime}\left(v_{i j}+\varepsilon v_{i j}\right)$ maximally by $\varepsilon \%$, which leads to $(1+\varepsilon) \cdot F_{i j}^{\prime \prime}\left(v_{i j}+\varepsilon v_{i j}\right)$. Therefore, the total increase rate of $F_{i j}^{\prime \prime}\left(v_{i j}\right)$ can be estimated by

$$
\begin{aligned}
\varepsilon^{\prime} & =\frac{(1+\varepsilon) F_{i j}^{\prime \prime}(v+\varepsilon v)-F_{i j}^{\prime \prime}(v)}{F_{i j}^{\prime \prime}(v)}=\frac{(1+\varepsilon)\left[\alpha(v+\varepsilon v)^{-1}+\beta(v+\varepsilon v)^{2}\right]-\left(\alpha v^{-1}+\beta v^{2}\right)}{\alpha v^{-1}+\beta v^{2}} \\
& =\frac{\left[(1+\varepsilon)^{3}-1\right]}{1+\frac{\alpha}{\beta v^{3}}}<\left[(1+\varepsilon)^{3}-1\right]=3 \varepsilon+3 \varepsilon^{2}+\varepsilon^{3} \approx 3 \varepsilon
\end{aligned}
$$

Thus, we obtain a loose upper limit, i.e., $3 \varepsilon \%$, for the increase rate of $F_{i j}^{\prime \prime}\left(v_{i j}\right)$ for a $\varepsilon \%$ speed increase. Note that 
the above upper limit is only for the first part of the FCR, whereas the other part, i.e., $\gamma+\varphi f_{i j}$, as well as the total travel time, is not affected; thus, the general increase rate of the whole objective value should be much lower than $3 \varepsilon \%$.

Based on Theorem 2 and the above deduction, we have the following proposition for gauging how far the solution of $\varepsilon$-CPRP is from the optimal solution of the original CPRP.

Proposition 3. After applying the post-optimization procedure in Fig. 5, an optimal $\pi$ solution of $\varepsilon$-CPRP with the upper speed limit $\bar{v}_{i j}(1+\varepsilon)$ is a feasible solution of the original CPRP if the travel speed $v_{i j}$ of any arc $(i, j)$ in $\pi$ does not exceed $\bar{v}_{i j}$ and has at most $3 \varepsilon \%$ higher objective function value than the optimal solution of CPRP in the worst case.

Thus, based on Propositions 1 and 3, the $\varepsilon$-CPRP model can be used to obtain either a feasibility-guaranteed solution under original upper speed limits or an optimality-gap-guaranteed solution by increasing the upper speed limits by $\varepsilon \%$. As discussed in the next experimental section, we let the upper speed limit $\bar{v}$ of all tested PRP instances increase by $\varepsilon \%$ such that all the obtained solutions will be only at most $3 \varepsilon \%$ above the optimal ones and their feasibilities can be judged by examining if their maximum travel speeds are under $\bar{v}$ or not. Even though in some instances the resulting travel speed may exceed the upper speed limit by $\varepsilon \%$ maximum, these solutions can still be considered as practically feasible because $\varepsilon$ is very small, e.g., 0.01 in our experiments.

\section{Computational experiments}

In this section, we present the results of computational experiments on several benchmark datasets for testing the proposed $\varepsilon-C P R P$ and $\varepsilon-C P R P^{\prime}$ models, and the obtained solutions are compared with the existing solutions in the literature. The MIP solver AMPL/CPLEX (version 12.6.0.1) was applied to solve benchmark problem instances. Computational experiments were conducted on a PC server with two $2.30 \mathrm{GHz}$ Intel ${ }^{\circledR}$ Xeon(R) CPUs and $110 \mathrm{~GB}$ memory. The approximation errors in all solutions obtained by the $\varepsilon-C P R P$ ( or $\varepsilon-C P R P^{\prime}$ ) model were eliminated by the post-optimization procedure given in Fig. 5. The time limit was set as $2 \mathrm{~h}$ for all test instances, and the relative MIP optimality gap (referred to as O.Gap) is used to indicate whether the problem was solved to optimality within the time limit $(O . G a p=0.0 \%)$ or not $(O . G a p>0.0 \%)$. The test problems are introduced in Section 6.1, and the experimental analysis and comparisons are presented in Subsections 6.2-6.4.

\subsection{Tested problem instances}

We used three sets of benchmark PRP instances in the experiments. The first set (UK-A) was generated by Demir et al. (2012) and is available at http://www.apollo.management.soton.ac.uk/prplib.htm, which includes real geographical distances between randomly selected cities in the UK, with random time windows, demands, and service time for customers. The second and third sets of PRP instances, noted as UK-B and UK-C, were provided by Kramer et al. (2015a) and are available at http://w1.cirrelt.ca/ vidalt/en/VRP-resources.html. The average distance between the nodes of these instances is approximately $80 \mathrm{~km}$, and the planning horizon is $9 \mathrm{~h}$. We solved all the instances of $10,15,20$, and 25 customers with each of the three objective functions given in Eq. (14). Thus, the total number of tested problem instances is 720 . Owing to the limited paper length, we provided only a portion of the results for analysis and illustration purposes. The complete and detailed results are available online at http://sites.psu.edu/auk3/CPRP. 
We used the same type of the 6.35-ton (curb weight) diesel truck vehicle used by Demir et al. (2012), Franceschetti et al. (2013), Kramer et al. (2015a, 2015b), and Fukasawa et al. (2016, 2017). Based on the truck parameters in these references, the coefficients of the fuel-speed/load function in Eq. (7) can be calculated as follows:

$$
\alpha=1.01763908 \times 10^{-3}, \quad \beta=1.41223439 \times 10^{-7}, \gamma=5.33605218 \times 10^{-5}, \varphi=8.40323178 \times 10^{-9}
$$

\subsection{Drivers' wage paid from beginning of time horizon (DPFB)}

First, we solved the problem instances with the objective function given in Eq. (14a), which includes fuel-related costs and drivers' wages paid from the beginning of the time horizon (DPFB). We solved a total of 240 PRP instances with $10,15,20$, and 25 customers in groups UK-A, UK-B, and UK-C by using the $\varepsilon-C P R P$ and $\varepsilon-C P R P^{\prime}$ with $\varepsilon=0.1 \%$ and $\varepsilon=0.01 \%$, respectively. The detailed results of all tested instances are available online at http://sites.psu.edu/auk3/CPRP. The results of the first five instances of 10, 20, and 25 customers were selected and presented in Tables 3 and 4, respectively. In Table 3, the subcolumn Cost $(\pi)$ indicates the cost obtained directly by the MILP model without using the post-optimization procedure, and the subcolumn Cost ( $\left.\pi^{*}\right)$ indicates the cost improved after the post-optimization procedure. It is observed that the average gaps between $\operatorname{Cost}(\pi)$ and $\operatorname{Cost}\left(\pi^{*}\right)$ are relatively small, ranging only from -0.001 to -0.075 with respect to different settings of model parameters, which indicates that the solution gaps are strictly controlled by parameter $\varepsilon$. According to Theorem 2 and Proposition 3, the obtained solutions are guaranteed to have a maximum deviation of $3 \varepsilon$. Note that for problem instance UK-B10-02, the solutions with $\varepsilon=0.1 \%$ have at least one arc with travel speed between $\bar{v}_{i j}$ and $(1+\varepsilon) \bar{v}_{i j}$, i.e., the upper speed limit is exceeded. The average computational time used for all instances of 10 customers takes only a few seconds in our PC server. It should be noted that for all tested instances of 10 customers, our solutions were very close to those by Demir et al. (2012), Kramer et al. (2015a), and Fukasawa et al. (2016, 2017).

Table 3 Experiment results for PRP instances of 10 customers under DPFB policy

\begin{tabular}{|c|c|c|c|c|c|c|c|c|c|c|}
\hline \multirow[b]{3}{*}{ Prob. No. } & \multirow{3}{*}{$\begin{array}{l}\text { Demir et al. } \\
\text { (2012) }\end{array}$} & \multirow{3}{*}{$\begin{array}{l}\text { Kramer et } \\
\text { al. (2015a) }\end{array}$} & \multicolumn{4}{|c|}{$\varepsilon=0.1 \%$} & \multicolumn{4}{|c|}{$\varepsilon=0.01 \%$} \\
\hline & & & \multicolumn{2}{|c|}{$\varepsilon-C P R P$} & \multicolumn{2}{|c|}{$\varepsilon-C P R P^{\prime}$} & \multicolumn{2}{|c|}{$\varepsilon-C P R P$} & \multicolumn{2}{|c|}{$\varepsilon-C P R P^{\prime}$} \\
\hline & & & Cost $(\pi)$ & Cost $\left(\pi^{*}\right)$ & Cost $(\pi)$ & $\operatorname{Cost}\left(\pi^{*}\right)$ & $\operatorname{Cost}(\pi)$ & $\operatorname{Cost}\left(\pi^{*}\right)$ & $\operatorname{Cost}(\pi)$ & Cost $\left(\pi^{*}\right)$ \\
\hline UK-A10-01 & 170.66 & 170.64 & 170.662 & 170.661 & 170.743 & 170.656 & 170.641 & 170.641 & 170.644 & 170.642 \\
\hline UK-A10-02 & 204.87 & 204.88 & 204.904 & 204.896 & 205.009 & 204.896 & 204.878 & 204.878 & 204.882 & 204.879 \\
\hline UK-A10-03 & 200.33 & 200.34 & 200.354 & 200.354 & 200.462 & 200.363 & 200.341 & 200.341 & 200.343 & 200.342 \\
\hline UK-A10-04 & 189.94 & 189.89 & 189.905 & 189.896 & 189.986 & 189.896 & 189.887 & 189.885 & 189.889 & 189.886 \\
\hline UK-A10-05 & 175.61 & 175.59 & 175.611 & 175.607 & 175.688 & 175.604 & 175.592 & 175.591 & 175.594 & 175.592 \\
\hline UK-B10-01 & -- & 246.45 & 246.490 & 246.474 & 246.554 & 246.500 & 246.449 & 246.447 & 246.450 & 246.447 \\
\hline UK- B10-02 & -- & 303.73 & $\underline{303.784}$ & $\underline{303.756}$ & $\underline{303.860}$ & $\underline{303.790}$ & 303.740 & 303.739 & 303.744 & 303.738 \\
\hline UK-B10-03 & -- & 301.89 & 301.908 & 301.905 & 301.988 & 301.949 & 301.894 & 301.893 & 301.895 & 301.893 \\
\hline UK-B10-04 & -- & 273.91 & 273.940 & 273.929 & 274.044 & 273.966 & 273.906 & 273.906 & 273.908 & 273.906 \\
\hline UK-B10-05 & -- & 255.08 & 255.147 & 255.131 & 255.227 & 255.146 & 255.083 & 255.080 & 255.084 & 255.079 \\
\hline UK-C10-01 & -- & 210.18 & 210.203 & 210.200 & 210.270 & 210.209 & 210.184 & 210.184 & 210.185 & 210.184 \\
\hline UK- C10-02 & -- & 271.93 & 271.945 & 271.945 & 272.069 & 271.965 & 271.933 & 271.932 & 271.936 & 271.932 \\
\hline UK-C10-03 & -- & 229.18 & 229.192 & 229.192 & 229.303 & 229.199 & 229.178 & 229.178 & 229.180 & 229.179 \\
\hline UK-C10-04 & -- & 230.59 & 230.542 & 230.531 & 230.609 & 230.575 & 230.521 & 230.521 & 230.522 & 230.521 \\
\hline
\end{tabular}




\begin{tabular}{|c|c|c|c|c|c|c|c|c|c|c|}
\hline UK-C10-05 & -- & 205.49 & 205.527 & 205.510 & 205.576 & 205.548 & 205.494 & 205.493 & 205.494 & 205.493 \\
\hline AVG gap & & & & -0.008 & & -0.075 & & -0.001 & & \\
\hline
\end{tabular}

Note: Italicized and underlined values indicate that the maximal travel speed exceeds the upper speed limit by at most $\varepsilon$.

In Table 4, the experimental results for the first 5 instances of PRP with 20 and 25 customers in UK-A, UK-B, and UK-C are presented and compared with the solutions by Fukasawa et al. (2016, 2017). In Table 4, column V.N. indicates the number of vehicles used in the solution, column T./O.Gap indicates the CPU time used if the solution was optimally solved, or the optimality gap if the problem could not be solved within the time limit, and column Imp.\% indicates the deviation of the solution from the best-known solution by Fukasawa et al. (2016, 2017). It can be observed that the new solutions have significant improvements compared to the solutions provided by Fukasawa et al. $(2016,2017)$. Note that for instances UK_B25_04 and UK_B25_05, the solutions have at least one arc with travel speed exceeding the upper speed limit by $\varepsilon \%$. From a theoretical point of view, these two instances are incomparable to the earlier results by Fukasawa et al. $(2016,2017)$. However, this slight relaxation on the upper speed limit is trivial from a practical point of view. In terms of computational efficiency, $\varepsilon$-CPRP with $\varepsilon=0.1 \%$ required longer CPU times than the algorithm by Fukasawa et al. (2017) but shorter CPU times than the algorithm by Fukasawa et al. (2016).

Table 4. Experiments and comparisons for PRP instances of 20 customers using DPFB policy

\begin{tabular}{|c|c|c|c|c|c|c|c|c|c|}
\hline \multirow{2}{*}{$\begin{array}{l}\text { Problem } \\
\text { Instances }\end{array}$} & \multicolumn{3}{|c|}{ Fukasawa et al. (2016) } & \multicolumn{2}{|c|}{ Fukasawa et al. (2017) } & \multicolumn{4}{|c|}{$\varepsilon$-CPRP $(\varepsilon=0.1 \%)$} \\
\hline & Cost & V.N. & Time (s) & Cost & Time (s) & Cost & V.N. & T./O.Gap & Imp.\% \\
\hline UK_A20_01 & 352.447 & 5 & 3600 & 351.82 & 13.9 & 323.208 & 3 & 334 & -8.13 \\
\hline UK_A20_02 & 365.767 & 5 & 3600 & 365.77 & 1.8 & 330.070 & 3 & 358 & -9.76 \\
\hline UK_A20_03 & 230.486 & 5 & 3600 & 230.49 & 11.8 & 207.664 & 3 & 54 & -9.90 \\
\hline UK_A20_04 & 347.043 & 5 & 3600 & 347.04 & 43.5 & 324.762 & 3 & 396 & -6.42 \\
\hline UK_A20_05 & 329.626 & 5 & 3600 & 323.44 & 16.6 & 295.559 & 3 & 271 & -8.62 \\
\hline UK_B20_01 & -- & -- & -- & 469.35 & 0.02 & 445.890 & 4 & 6 & -5.00 \\
\hline UK_B20_02 & -- & -- & -- & 477.05 & 0.02 & 440.503 & 3 & 11 & -7.66 \\
\hline UK_B20_03 & -- & -- & -- & 354.46 & 0.03 & 324.020 & 3 & 20 & -8.59 \\
\hline UK_B20_04 & -- & -- & -- & 523.59 & 0.1 & 473.428 & 3 & 9 & -9.58 \\
\hline UK_B20_05 & -- & -- & -- & 447.33 & 1.2 & 430.525 & 4 & 22 & -3.76 \\
\hline UK_C20_01 & -- & -- & -- & 432.82 & 90.3 & 375.455 & 3 & 35 & -13.25 \\
\hline UK_C20_02 & -- & -- & -- & 448.29 & 4.4 & 385.713 & 3 & 489 & -13.96 \\
\hline UK_C20_03 & -- & -- & -- & 287.04 & 4.1 & 263.840 & 3 & 382 & -8.08 \\
\hline UK_C20_04 & -- & -- & -- & 434.23 & 4.5 & 380.938 & 3 & 225 & -12.27 \\
\hline UK_C20_05 & -- & -- & -- & 381.7 & 5.9 & 344.829 & 3 & 152 & -9.66 \\
\hline UK_A25_01 & -- & -- & -- & 389.46 & 3600 & 282.149 & 3 & 718 & -27.55 \\
\hline UK_A25_02 & -- & -- & -- & 373.92 & 3600 & 345.050 & 4 & $0.6 \%$ & -7.72 \\
\hline UK_A25_03 & -- & -- & -- & 273.08 & 3600 & 217.996 & 3 & 5403 & -18.95 \\
\hline UK_A25_04 & -- & -- & -- & 321.14 & 3600 & 264.075 & 3 & $3.8 \%$ & -18.96 \\
\hline UK_A25_05 & -- & -- & -- & 365.00 & 2925 & 329.146 & 4 & 1564 & -9.82 \\
\hline UK_B25_01 & 452.09 & 5 & 29.4 & 474.35 & 1 & 414.519 & 3 & 34 & -8.31 \\
\hline UK_B25_02 & 497.98 & 5 & 710.2 & 530.00 & 10.6 & 471.362 & 4 & 610 & -5.35 \\
\hline UK_B25_03 & 367.15 & 5 & 39.8 & 390.17 & 2.6 & 332.807 & 3 & 769 & -9.35 \\
\hline UK_B25_04 & 438.75 & 5 & 36.4 & 458.56 & 132 & $\underline{404.904}$ & 3 & 332 & -7.71 \\
\hline
\end{tabular}




$\begin{array}{lccccccccc}\text { UK_B25_05 } & 462.64 & 5 & 36.5 & 489.16 & 11.2 & \underline{\mathbf{4 4 0 . 4 4 6}} & 4 & 920 & -4.80 \\ \text { UK_C25_01 } & -- & -- & -- & 416.88 & 163 & \mathbf{3 7 6 . 3 0 9} & 3 & 6.7 \% & -10.05 \\ \text { UK_C25_02 } & -- & -- & -- & 467.51 & 548 & \mathbf{4 4 0 . 4 7 0} & 4 & 8.4 \% & -6.49 \\ \text { UK_C25_03 } & -- & -- & -- & 326.31 & 3600 & \mathbf{2 8 9 . 1 4 0} & 3 & 10.3 \% & -11.94 \\ \text { UK_C25_04 } & -- & -- & -- & 386.21 & 3600 & \mathbf{3 3 2 . 9 5 5} & 3 & 6620 & -13.79 \\ \text { UK_C25_05 } & -- & -- & -- & 458.55 & 3600 & \mathbf{4 1 8 . 5 6 6} & 4 & 7.3 \% & -9.50\end{array}$

Note: Italicized and underlined values indicate that the maximal travel speed exceeds the upper speed limit by at most $\varepsilon$, and boldfaced values indicate new best-known solutions.

Finally, we tested the performance of the $\varepsilon$-CPRP model with respect to different values of parameter $\varepsilon$. Twenty instances of UK-A20 were solved with $\varepsilon=2 \%, 1 \%, 0.5 \%, 0.1 \%, 0.05 \%$, and $0.01 \%$. In Fig. 6, the average computational time and the average deviations of objective values from those obtained with $\varepsilon=0.01 \%$ are plotted against the values of parameter $\varepsilon$. It can be observed that the computational time increases from $140 \mathrm{~s}$ to $1050 \mathrm{~s}$ as parameter $\varepsilon$ decreases from $2 \%$ to $0.01 \%$. Meanwhile, the solution accuracy has been improved from $0.32 \%$ to $0.00 \%$. This graph suggests that an ideal trade-off value for parameter $\varepsilon$ for these problems should be approximately $0.1 \%$, where the solution accuracy is very close to the best ones obtained by $\varepsilon=0.01 \%$ while the computational time does not increase significantly.

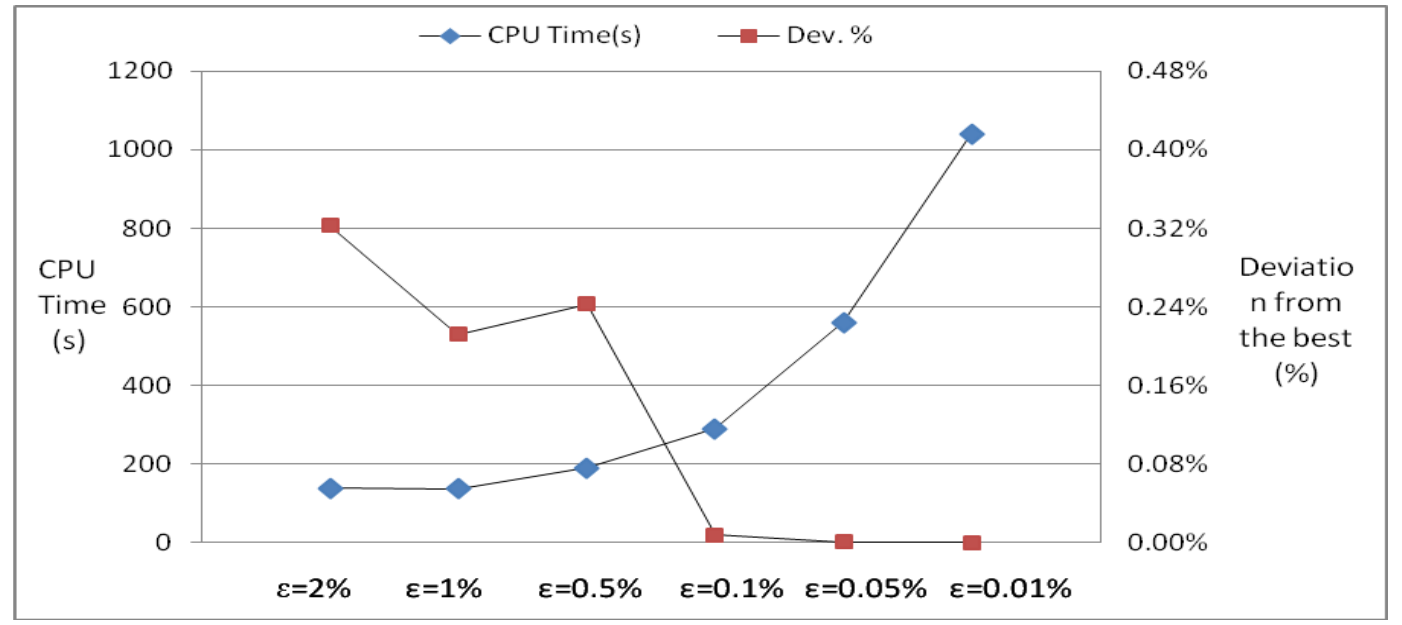

Fig. 6 Trends of solution accuracy and CPU time against parameter $\varepsilon$ for instances in UK_A20

\subsection{Drivers' wage paid from the departure time (DPFD)}

Next, we tested the $\varepsilon$-CPRP model with the objective function in Eq. (14b), which includes the fuel-related cost and the drivers' wage calculated from the departure time (DPFD). Under the DPFD policy, the departure time from depot can be scheduled to shorten the total travel time. We first solved the 60 PRP instances with 10 customers by $\varepsilon-C P R P$ and $\varepsilon-C P R P^{\prime}$ with $\varepsilon=0.01 \%$. In Table 5, the solutions of the first five instances were compared to the existing solutions by Kramer et al. (2015b) and Fukasawa et al. (2016). Next, we solved the 180 PRP instances with 15,20 , and 25 customers using $\varepsilon-C P R P$ with $\varepsilon=0.1 \%$ and summarized the results in Table 6 .

It can be observed from Table 5 that our solutions are very close to the best ones in the study by Kramer et al. (2015b) and Fukasawa et al. (2016). Some of the optimal solutions reported by Fukasawa et al. (2016), e.g., for instances UK_B10_02, UK_B10_03, UK_B10_04, UK_B10_05, UK_C10_02, and UK_C10_03, are not shown to be optimal in our experiments. There are also other five solutions, i.e., UK_A10_03, UK_B10_03, UK_B10_04, UK_B10_05, and UK_C10_03, that had been updated with new best solutions. Note that the new solutions did not violate the upper speed limit; hence, they are feasible. In terms of computational efficiency, $\varepsilon-C P R P$ and $\varepsilon-C P R P$ 
used only a few seconds for the tested instances of 10 customers. The heuristic algorithm given by Kramer et al. (2015b) is the best, which used less than 1 s. The MICP algorithm by Fukasawa et al. (2016) had high efficiencies for the instances in UK_B (less than $1 \mathrm{~s}$ ) but low efficiencies for the instances in UK_A (longer than $1 \mathrm{~h}$ ).

Table 6 provides the summary of the results for 180 UK_PRP instances with 15, 20, and 25 customers. It can be observed that 171 out of 180 instances were solved to optimality within the time limit, and the final solutions were feasible in 166 out of 180 instances with respect to the maximum speed limit. The results show that the tightness of customers' time windows has a significant effect on the solution time. Instances in UK_B, which have tighter time windows than those in UK_A and UK_C, were solved in much shorter times compared to those in UK_A and UK_C. Instances with tighter time windows also had relatively higher costs and used a higher number of vehicles in the solutions.

Table 5. Result comparisons for UK-A10, UK-B10, and UK-C10 under DPFD policy $(\varepsilon=0.01 \%)$

\begin{tabular}{|c|c|c|c|c|c|c|c|c|c|}
\hline \multirow{2}{*}{$\begin{array}{c}\text { Problem } \\
\text { ID }\end{array}$} & \multirow{2}{*}{$\begin{array}{c}\text { Kramer et al. } \\
(2015 b)\end{array}$} & \multicolumn{2}{|c|}{ Fukasawa et al. (2016) } & \multicolumn{3}{|c|}{$\varepsilon-C P R P(\varepsilon=0.01 \%)$} & \multicolumn{3}{|c|}{$\varepsilon-C P R P^{\prime}(\varepsilon=0.01 \%)$} \\
\hline & & Cost & Time (s) & Cost & Time (s) & Imp. $\%$ & Cost & Time (s) & Imp. $\%$ \\
\hline UK_A10_01 & 169.04 & 168.820 & 3600 & 168.821 & 14 & 0.00 & 168.822 & 8 & 0.00 \\
\hline UK_A10_02 & 203.82 & 203.824 & 3600 & 203.825 & 12 & 0.00 & 203.826 & 8 & 0.00 \\
\hline UK_A10_03 & 198.50 & 198.351 & 3600 & 198.271 & 19 & -0.04 & 198.272 & 10 & -0.04 \\
\hline UK_A10_04 & 187.29 & 186.594 & 3600 & 186.594 & 6 & 0.00 & 186.595 & 5 & 0.00 \\
\hline UK_A10_05 & 172.89 & 172.894 & 3600 & 172.894 & 12 & 0.00 & 172.895 & 9 & 0.00 \\
\hline UK_B10_01 & 217.96 & 217.959 & 0.3 & 217.962 & 2 & 0.00 & 217.961 & 1 & 0.00 \\
\hline UK_B10_02 & 263.86 & 266.738 & 0.2 & 263.861 & 1 & 0.00 & 263.861 & 1 & 0.00 \\
\hline UK_B10_03 & 261.50 & 261.496 & 0.2 & 260.056 & 2 & -0.55 & 260.057 & 1 & -0.55 \\
\hline UK_B10_04 & 256.78 & 256.779 & 0.2 & 251.289 & 3 & -2.14 & 251.290 & 2 & -2.14 \\
\hline UK_B10_05 & 253.53 & 254.529 & 0.1 & 248.328 & 3 & -2.05 & 248.328 & 2 & -2.05 \\
\hline UK_C10_01 & 171.96 & 171.956 & 51.2 & 171.957 & 8 & 0.00 & 171.957 & 4 & 0.00 \\
\hline UK_C10_02 & 219.37 & 230.525 & 85.5 & 219.370 & 9 & 0.00 & 219.371 & 6 & 0.00 \\
\hline UK_C10_03 & 204.97 & 204.972 & 24.7 & 199.945 & 3 & -2.45 & 199.946 & 1 & -2.45 \\
\hline UK_C10_04 & 187.88 & 187.883 & 13.2 & 187.883 & 3 & 0.00 & 187.884 & 2 & 0.00 \\
\hline UK_C10_05 & 184.11 & 184.098 & 89.1 & 184.098 & 7 & 0.00 & 184.099 & 5 & 0.00 \\
\hline $\mathrm{AVG}$ & & & & & & -0.48 & & & -0.48 \\
\hline
\end{tabular}

Note: Boldfaced numbers indicate the best-known values whereas italicized ones indicate new best-known values.

Table 6. Summary of results of $\varepsilon$-CPRP for PRP instances with 15,20 , and 25 customers under DPFD policy $(\varepsilon=0.1 \%)$

\begin{tabular}{cccccccc}
\hline Prob. Set & Ins. Num. & AVG Cost & AVG V.N. & AVG CPU Time (s) & AVG O.Gap \% & Optimality & Exc. SPD \\
\hline UK_A15 & 20 & 247.847 & 2.6 & 29.3 & $0.0 \%$ & $20 / 20$ & $0 / 20$ \\
UK_B15 & 20 & 325.145 & 3.6 & 2.7 & $0.0 \%$ & $20 / 20$ & $1 / 20$ \\
UK_C15 & 20 & 271.339 & 2.9 & 13.4 & $0.0 \%$ & $20 / 20$ & $3 / 20$ \\
UK_A20 & 20 & 314.445 & 3.1 & 546 & $0.0 \%$ & $20 / 20$ & $0 / 20$ \\
UK_B20 & 20 & 414.240 & 4.3 & 11.1 & $0.0 \%$ & $20 / 20$ & $1 / 20$ \\
UK_C20 & 20 & 347.985 & 3.5 & 563 & $0.0 \%$ & $20 / 20$ & $4 / 20$ \\
UK_A25 & 20 & 333.425 & 3.8 & 3695 & $0.7 \%$ & $15 / 20$ & $2 / 20$ \\
UK_B25 & 20 & 434.206 & 4.6 & 233 & $0.0 \%$ & $20 / 20$ & $3 / 20$ \\
UK_C25 & 20 & 366.584 & 4.0 & 3581 & $1.2 \%$ & $16 / 20$ & $0 / 20$ \\
\hline
\end{tabular}




\section{Conclusion}

This study investigated the CPRP, an extension of the discrete PRP, which aims at identifying a set of continuously optimized routes and schedules for a fleet of fossil fuel-powered vehicles in the context of VRPTW. The proposed $\varepsilon$-CPRP model extended the existing discrete PRP models on three aspects: (1) the travel speed, travel time, load, and FCR of a vehicle over each arc, as well as the departure time, arrival time, and waiting time upon each node, are all treated as continuous decision variables and optimized synchronously; (2) solution of the $\varepsilon$-CPRP model is guaranteed to be practically feasible and as close as necessary to the theoretically optimal solution; and (3) the $\varepsilon$-CPRP model is computationally efficient (up to 25 customers). Comparative experiments showed that the $\varepsilon$-CPRP model could yield better solutions than existing discrete PRP models, and many of the UK_PRP instances were updated with new best solutions in our computational experiments.

It is worth mentioning that, by using the iterative partial optimization (IPO) strategy for vehicle routing problems formulated with MILP models (see Xiao et al., 2016), a fix-and-optimize heuristic algorithm can be developed based on the $\varepsilon$-CPRP model to solve large-sized PRP instances with near-optimal solutions and controllable CPU time. This indicates the proposed theoretical-like $\varepsilon$-CPRP model has a practical value in large-scale applications as well. In particularly, the linearization method for the time-speed relationship is also applicable to other types of VRPs involving travel speed and travel time optimization, and the linearization methods for non-FCR functions can also help to bound a linear relationship between the fuel consumption and speed for other variants of PRP.

\section{Acknowledgements}

This work is partly supported by the National Natural Science Foundation of China under Grants No. 71871003 and No. 61376042.

\section{Reference}

1. Barth M. J., Boriboonsomsin K., 2008. Real-world CO2 impacts of traffic congestion. Transportation Research Record: Journal of the Transportation Research Board 2058, 163-171.

2. Barth M. J., Younglove T., Scora G., 2005. Development of a heavy-duty diesel modal emissions and fuel consumption model. Technical Report. UCB-ITSPRR-2005-1, California PATH Program, Institute of Transportation Studies, University of California at Berkeley.

3. Bektas T., Laporte G., 2011. The Pollution-Routing Problem. Transportation Research Part B 45 (2011), 1232-1250.

4. Castillo I., Westerlund T., 2005. An $\varepsilon$-accurate model for optimal unequal area facility layout. Computers \& Operations Research. $32,429-447$

5. Dabia S., Demir E., Van Woensel T., 2017. An Exact Approach for a Variant of the Pollution-Routing Problem. Transportation Science 51(2), 607-628.

6. Demir E., Bektas T., Laporte G., (2012). An adaptive large neighborhood search heuristic for the Pollution-Routing Problem. European Journal of Operational Research 223 (2012) 346-359.

7. Demir E., Bektas T., Laporte G., 2011. A comparative analysis of several vehicle emission models for road freight transportation. Transportation Research Part D 6, 347-357.

8. Demir E., Bektas T., Laporte G., 2014a. A review of recent research on green road freight transportation. European Journal of Operational Research 237(3), 775-793.

9. Demir E., Bektas T., Laporte G., 2014b. The bi-objective Pollution-Routing Problem, European Journal of Operational Research 232 (3), 464-478.

10. Erdogan S., Miller-Hooks E., 2012. A green vehicle routing problem. Transportation Research Part E: Logistics. Transp. Rev. 48, $100-114$. 
11. Eshtehadi R., Fathian M., Demir E. (2017). Robust solutions to the pollution-routing problem with demand and travel time uncertainty. Transportation Research Part D 51, 351-363.

12. Figliozzi M. A., 2010. Vehicle routing problem for emissions minimization. Transportation Research Record: Journal of the Transportation Research Board 2197, 1-7.

13. Franceschetti A., Demir E., Honhon D., Woensel T. V., Laporte G., Stobbe M., 2017. A metaheuristic for the time-dependent pollution-routing problem. European Journal of Operational Research 259, 972-991.

14. Franceschetti A., Honhon D., Woensel T. V., Bektas T., Laporte G., 2013. The time-dependent pollution-routing problem. Transportation Research Part B 56 (2013), 265-293.

15. Fukasawa R., He Q., Santosz F., Song Y, 2017. A Joint Routing and Speed Optimization Problem. Available at https://web.stanford.edu/ boyd/papers/pdf/cvx_pwl_fit.pdf. [Accessed at Nov 20, 2017].

16. Fukasawa R., He Q., Song Y., 2016. A disjunctive convex programming approach to the pollution-routing problem. Transportation Research Part B 94, 61-79.

17. Gaur D. R., Mudgal A., Singh R. R., 2013. Routing vehicles to minimize fuel consumption. Oper. Res. Lett. 41, 576-580.

18. Hickman A. J., 1999. Methodology for calculating transport emissions and energy consumption. TRANSPORT RESEARCH LABORATORY, pp.69-73.

19. International Energy Agency (IEA), 2016a. CO2 emissions from fuel combustion-Highlight. https://www.iea.org/publications/ freepublications/publication/co2-emissions-from-fuel-combustion-highlights-2016.html. <accessed 2017-07-08>.

20. International Energy Agency (IEA), 2016b. Global EV Outlook 2016. https://www.iea.org/publications/freepublications/ publication/Global_EV_Outlook_2016.pdf. <accessed 2017-07-08>.

21. Jabali O., Van Woensel T., Kok A. G. de, 2012. Analysis of travel times and CO2 emissions in time-dependent vehicle routing. Production and Operations Management 21(6), 1060-1074.

22. Konak A., Kulturel-Konak S., Norman B.-A., Smith A. E., 2006. A new mixed integer programming formulation for facility layout design using flexible bays. Oper. Res. Lett. 34, 660-672 .

23. Kara I., Kara B. Y., Yetis M. K., 2007. Energy minimizing vehicle routing problem. In: Dress A, Xu Y, Zhu B, editors. Combinatorial Optimization and Applications: FIRST International Conference, LNCS 4616. Berlin/Heidelberg: Springer; 2007. p. $62-71$.

24. Kara I., Kara B. Y., Yetis M. K., 2008. Vehicle routing problem. In C. Tonic, \& G. Hrvoje (Eds.), Commulative vehicle routing problems (pp.85-98). In Tech.

25. Koc C., Bektas T., Jabali O., Laporte G., 2014. The fleet size and mix pollution-routing problem. Transportation Research Part B, 70, 239-254.

26. Koç C., Karaoglan I., 2016. The green vehicle routing problem: A heuristic based exact solution approach. Applied Soft Computing 39, 154-164.

27. Kramer R., Maculan N., Subramanian A., Vidal T., 2015b. A speed and departure time optimization algorithm for the pollution-routing problem. European Journal of Operational Research, 247, 782-787.

28. Kramer R., Subramanian A., Vidal T., Cabral L. dos A. F., 2015a. A matheuristic approach for the Pollution-Routing Problem. European Journal of Operational Research 243, 523-539.

29. Kuo Y., 2010. Using simulated annealing to minimize fuel consumption for the time-dependent vehicle routing problem. Computers \& Industrial Engineering 59, 157-165.

30. Leggieri V., Haouari M., 2017. A practical solution approach for the green vehicle routing problem. Transportation Research Part E 104, 97-112.

31. Lin C., Choy K., Ho G., Chung S., Lam H., 2014. Survey of green vehicle routing problem: Past and future trends. Expert Systems with Applications,41(4),1118-1138.

32. Saka O. C., Gurel S., Woensel T. V., 2017. Using cost change estimates in a local search heuristics for the pollution routing problem. OR Spectrum 39, 557-587.

33. Schneider M., Stenger A., Goeke D., 2014. The electric vehicle-routing problem with time windows and recharging stations. 
Transportation Science 48, 500-520.

34. Sherali H.D., Fraticelli B.M.P., Meller, R.D., 2003. Enhanced model formulations for optimal facility layout. Oper. Res. 51, 629-644.

35. Suzuki Y., 2016. A dual-objective metaheuristic approach to solve practical pollution routing problem. Int. J. Production Economics 176, 143-153.

36. Tajik N., Tavakkoli-Moghaddam R., Vahdani B., Mousavi S. M., 2014. A robust optimization approach for pollution routing problem with pickup and delivery under uncertainty. Journal of Manufacturing Systems. Journal of Manufacturing Systems 33, $277-286$.

37. U.S. Energy Information Administration (EIA), 2017. Energy used for transportation - Energy Explained. https://www.eia.gov/ energyexplained/?page=use_energy_transportation. $<$ accessed 2017-07-08>.

38. Wang S., Meng Q., 2012. Sailing speed optimization for container ships in a liner shipping network. Transportation Research Part E 48, 701-714.

39. Xiao Y., Konak A., 2015. A simulating annealing algorithm to solve the green vehicle routing \& scheduling problem with hierarchical objectives and weighted tardiness. Applied Soft Computing 34, 372-388.

40. Xiao Y., Konak A., 2016. The heterogeneous green vehicle routing and scheduling problem with time-varying traffic congestion. Transportation Research Part E: Logistics and Transportation Review 88, 146-166.

41. Xiao Y., Konak A., 2017. A genetic algorithm with exact dynamic programming for the green vehicle routing \& scheduling problem. Journal of Clean Production 167, 1450-1463.

42. Xiao Y., Xie Y., Konak A., Kulturel-Konak S., 2017. A problem evolution algorithm with linear programming for the dynamic facility layout problem - A general layout formulation. Computers and Operations Research 88, 187-207.

43. Xiao Y., Zhao Q., Kaku I., Xu Y., 2012. Development of a Fuel Consumption Optimization Model for the Capacitated Vehicle Routing Problem. Computers \& Operations Research 39 (7), 1419-1431.

44. Xie Y., Zhou S., Xiao Y., Kulturel-Konak S., Konak A., 2018. A $\beta$-accurate linearization method of Euclidean distance for the facility layout problem with heterogeneous distance metrics. European Journal of Operational Research 265(1), 26-38.

45. Zhang J., Zhao Y., Xue W., Li j., 2015. Vehicle routing problem with fuel consumption and carbon emission. Int. J. Production Economics 170, 234-242.

\section{Appendix}

Table A1. parameters of vehicle used in Demir et al.(2012)

\begin{tabular}{lll}
\hline Notation & Description & Value \\
\hline$\xi$ & Fuel-to-air mass ratio & 1 \\
$\kappa$ & Heating value of a typical diesel fuel $(\mathrm{kJ} / \mathrm{g})$ & 44 \\
$\psi$ & Conversion factor $(\mathrm{g} / \mathrm{l})$ & 737 \\
$k$ & Engine friction factor $(\mathrm{KJ} / \mathrm{rev} / \mathrm{l})$ & 0.2 \\
$N_{e}$ & Engine speed (rev/s) & 33 \\
$V$ & Engine displacement $(\mathrm{l})$ & 5 \\
$\rho$ & Air density $\left(\mathrm{kg} / \mathrm{m}^{3}\right)$ & 1.2041 \\
$A$ & Frontal surface area $\left(\mathrm{m}^{2}\right)$ & 3.912 \\
$\mu$ & Curb-weight $(\mathrm{kg})$ & 6350 \\
$g$ & Gravitational constant (m/ $\left.\mathrm{s}^{2}\right)$ & 9.81 \\
$\phi$ & Road angle & 0 \\
$C_{d}$ & Coefficient of aerodynamic drag & 0.7 \\
$C_{r}$ & Coefficient of rolling resistance & 0.01 \\
$\varepsilon$ & Vehicle driver train efficiency & 0.4 \\
$\varpi$ & Efficiency parameter for diesel engines & 0.9 \\
\hline
\end{tabular}




\begin{tabular}{lll}
\hline$f_{c}$ & Fuel price per liter $(\mathfrak{f})$ & 1.4 \\
$d_{c}$ & Driver wage $(\mathfrak{f} / \mathrm{s})$ & 0.0022 \\
$v_{\min }$ & Lower speed limit $(\mathrm{m} / \mathrm{s})$ & 5.5 \\
$v_{\max }$ & Upper speed limit $(\mathrm{m} / \mathrm{s})$ & 25 \\
\hline
\end{tabular}

\section{Detailed derivation for Eq.(2) and Eq.(3).}

For a given arc $(i, j)$ with distance $D_{i j}$, the travel time $t$ is a function of average travel speed $v$ that can be expressed as a curve $t(v)=D_{i j} / v$. For a pair of two points $A\left(t_{0}, v_{0}\right)$ and $B\left(t_{1}, v_{1}\right)$ on the curve $t(v)=D_{i j} / v, \quad v_{0}<v_{1}$, the equation of line A-B can be written as $t=\frac{-D_{i j}}{v_{1} v_{0}} v+\left(\frac{D_{i j}}{v_{1}}+\frac{D_{i j}}{v_{0}}\right)$. For a given a speed $v$, the actual travel time $t$ is $D_{i j} / v$ while the approximate travel time by the line A-B would be $t^{\prime}=\frac{-D_{i j}}{v_{1} v_{0}} v+\left(\frac{D_{i j}}{v_{1}}+\frac{D_{i j}}{v_{0}}\right)$. The deviation of $t^{\prime}$ from $t$ can be written as a function of $v$ as follows.

$$
d(v)=\frac{\left(t^{\prime}-t\right)}{t}=\frac{\left[\frac{-D_{i j}}{v_{1} v_{0}} v+\left(\frac{D_{i j}}{v_{1}}+\frac{D_{i j}}{v_{0}}\right)-\frac{D_{i j}}{v}\right]}{\frac{D_{i j}}{v}}=-\frac{1}{v_{1} v_{0}} v^{2}+\frac{\left(v_{0}+v_{1}\right)}{v_{1} v_{0}} v-1
$$

Let the derivation of $d(v)$ be zero, i.e., $d^{\prime}(v)=0$, such that we have $d^{\prime}(v)=-\frac{2 v}{v_{1} v_{0}}+\frac{\left(v_{0}+v_{1}\right)}{v_{1} v_{0}}=0$ which leads to $v=\frac{1}{2}\left(v_{0}+v_{1}\right)$, indicating that the deviation function $d(v)$ has a maximum value of $\frac{\left(v_{0}-v_{1}\right)^{2}}{4 v_{1} v_{0}}$ at $v=\frac{1}{2}\left(v_{0}+v_{1}\right)$.

Let the maximum deviation value to be $\varepsilon$, i.e., $\frac{\left(v_{0}-v_{1}\right)^{2}}{4 v_{1} v_{0}}=\varepsilon$, such that we have $v_{1}^{2}-(4 \varepsilon+2) v_{0} v_{1}+v_{0} v_{0}=0$ which leads to $v_{1}=\left(1+2 \varepsilon+2 \sqrt{\varepsilon+\varepsilon^{2}}\right) v_{0}$. Let $\mu=1+2 \varepsilon+2 \sqrt{\varepsilon+\varepsilon^{2}}$, such that we have

$$
v_{1}=\mu v_{0} \text { and } t_{1}=\frac{D_{i j}}{\mu v_{0}},
$$

which means that the farthest point $\mathrm{B}$ on the curve $t=D_{i j} / v$ from point $\mathrm{A}\left(v_{0}, \frac{D_{i j}}{v_{0}}\right)$ is $\left(\mu v_{0}, \frac{D_{i j}}{\mu v_{0}}\right)$ such that for any point $\left(v, t^{\prime}\right)$ on the line A-B, the deviation calculated by Eq.(A1) does not exceed the given parameter $\varepsilon$. Similarly, we can continue to deduce $\left(t_{2}, v_{2}\right)$ starting from $\left(t_{1}, v_{1}\right),\left(t_{3}, v_{3}\right)$ from $\left(t_{2}, v_{2}\right), \ldots$, and $\left(t_{\eta}, v_{\eta}\right)$ from $\left(t_{\eta-1}, v_{\eta-1}\right)$, until $v_{\eta} \geq v_{\max }$ is satisfied. Thus, let $v_{0}=v_{\min }$, we find the recursive equations in Eq.(A3) and Eq.(A4) as follows.

$$
\begin{array}{ll} 
\begin{cases}v_{1}=\mu v_{\min }, t_{1}=\frac{1}{\mu v_{\min }} D_{i j} & \forall p=1 \\
v_{p}=\mu v_{p-1}, t_{p}=\frac{1}{\mu v_{p-1}} D_{i j} & \forall p=2,3, \ldots, \eta\end{cases} \\
\left\{\begin{array}{l}
v_{p}=\mu^{p} v_{\min } \\
t_{p}=D_{i j} /\left(\mu^{p} v_{\min }\right)
\end{array}\right. & \forall p=1,2, \ldots, \eta
\end{array}
$$


Let $v_{p}=\mu^{p} v_{\min } \geq v_{\max }$, we can transform Eq.(A4) into the following equation that calculates the minimum number of secant lines from $v_{\min }$ to $v_{\max }$ as follows.

$$
\eta=\left\lceil\frac{\ln v_{\text {max }}-\ln v_{\min }}{\ln \left(1+2 \varepsilon+2 \sqrt{\varepsilon+\varepsilon^{2}}\right)}\right\rceil,
$$

where $\lceil\bullet\rceil$ indicates the smallest integer larger than $\bullet$. Thus, there are $\eta$ secant lines, indexed by $p=1,2, \ldots ., \eta$, and the $p^{\text {th }}$ line can be written as $t=K_{p} v+B_{p}$ where $K_{p}$ and $B_{p}$ are the slope and intercept respectively, calculated by the following equations.

$$
\left\{\begin{array}{l}
K_{p}=\frac{t_{p}-t_{p-1}}{v_{p}-v_{p-1}}=\frac{D_{i j} /\left(\mu^{p} v_{\min }\right)-D_{i j} /\left(\mu^{p-1} v_{\min }\right)}{\mu^{p} v_{\min }-\mu^{p-1} v_{\min }}=-\frac{1}{\mu^{2 p-1} v_{\min }^{2}} D_{i j} \quad p=1,2, \ldots, \eta \\
B_{p}=t_{p}-K_{p} v_{p}=D_{i j} /\left(\mu^{p} v_{\min }\right)-\left(-\frac{1}{\mu^{2 p-1} v_{\min }^{2}} D_{i j}\right) \mu^{p} v_{\min }=\frac{1+\mu}{\mu^{p} v_{\min }} D_{i j}
\end{array}\right.
$$

\section{Detailed derivation for Eq.(10) and Eq.(11).}

For a pair of two points $A\left(x_{0}, y_{0}\right)$ and $B\left(x_{1}, y_{1}\right)$ on curve $y=x^{2}, x_{0}<x_{1}$, the equation of line A-B can be written as $y=\left(x_{1}+x_{0}\right) x-x_{1} x_{0}$. For a given a point $(x, y)$ on curve $y=x^{2}$ and the surrogate point $\left(x, y^{\prime}\right)$ by line A-B, where $y^{\prime}=\left(x_{1}+x_{0}\right) x-x_{1} x_{0}$, the deviation of $y^{\prime}$ from $y$ can be written as a function of $x$ as follows.

$$
d(x)=\frac{y^{\prime}-y}{y}=\frac{\left(x_{1}+x_{0}\right) x-x_{1} x_{0}-x x}{x x}=\left(x_{1}+x_{0}\right) x^{-1}-x_{1} x_{0} x^{-2}-1
$$

By setting derivation of $d(x)$ to zero, i.e., $d^{\prime}(x)=0$, we have $d^{\prime}(x)=-\left(x_{1}+x_{0}\right) x^{-2}+2 x_{1} x_{0} x^{-3}=0$ which leads to $x=\frac{2 x_{1} x_{0}}{x_{1}+x_{0}}$, indicating that function $d(x)$ has a maximum value of $\frac{\left(x_{1}-x_{0}\right)^{2}}{4 x_{0} x_{1}}$ at $x=\frac{2 x_{1} x_{0}}{x_{1}+x_{0}}$.

By letting the maximum deviation value be $\varepsilon$, i.e., $\frac{\left(x_{1}-x_{0}\right)^{2}}{4 x_{1} x_{0}}=\varepsilon$, we obtain $x_{1}^{2}-(4 \varepsilon+2) x_{0} x_{1}+x_{0}^{2}=0$ which leads to $x_{1}=\left(1+2 \varepsilon+2 \sqrt{\varepsilon+\varepsilon^{2}}\right) x_{0}$. Let $\mu=1+2 \varepsilon+2 \sqrt{\varepsilon+\varepsilon^{2}}$, such that we have

$$
x_{1}=\mu x_{0} \text { and } y_{1}=\left(\mu v_{0}\right)^{2} \text {, }
$$

which means that the farthest point $\mathrm{B}$ on curve $y=x^{2}$ from point $\mathrm{A}\left(x_{0}, x_{0}^{2}\right)$ is $\left(\mu x_{0},\left(\mu x_{0}\right)^{2}\right)$, such that for any point $\left(x, y^{\prime}\right)$ on the line A-B, the deviation calculated by Eq.(A7) does not exceed $\varepsilon$. Similarly, we can continue to deduce $\left(x_{2}, y_{2}\right)$ based on $\left(x_{1}, y_{1}\right),\left(x_{3}, y_{3}\right)$ based on $\left(x_{2}, y_{2}\right), \ldots$, and $\left(x_{\eta}, y_{\eta}\right)$ based on $\left(x_{\eta-1}, y_{\eta-1}\right)$, until $x_{\eta} \geq v_{\max }$ is satisfied. Thus, let $x_{0}=v_{\min }$, we get the following recursive equations in Eq.(A9) and Eq.(A10).

$$
\begin{aligned}
& \begin{cases}x_{1}=\mu x_{\text {min }}, y_{1}=\mu^{2} v_{\text {min }}^{2} & \forall p=1 \\
x_{p}=\mu x_{p-1}, y_{p}=\mu^{2} v_{p-1}^{2} & \forall p=2,3, \ldots, \eta\end{cases} \\
& \left\{\begin{array}{l}
x_{p}=\mu^{p} v_{\min } \\
y_{p}=\mu^{2 p} v_{\min }^{2}
\end{array} \quad \forall p=1,2, \ldots, \eta\right.
\end{aligned}
$$

Let $x_{p}=\mu^{p} x_{\min } \geq v_{\max }$, we can obtain the same expression as Eq.(A4) that calculates the minimum number of 
secant lines from $v_{\min }$ to $v_{\max }$. Thus, there are totally $\eta$ secant lines indexed by $p=1,2, \ldots, \eta$, and the $p^{\text {th }}$ line can be written as a general equation $t=b_{p} v+b_{p}$, where $k_{p}$ and $b_{p}$ are the slope and intercept respectively, calculated by the following equations.

$$
\left\{\begin{array}{l}
k_{p}=\frac{y_{p}-y_{p-1}}{x_{p}-x_{p-1}}=\frac{\left(\mu^{p} v_{\min }\right)^{2}-\left(\mu^{p-1} v_{\min }\right)^{2}}{\mu^{p} v_{\min }-\mu^{p-1} v_{\min }}=(\mu+1) \mu^{p-1} v_{\min } \\
b_{p}=y_{p}-k_{p} x_{p}=\left(\mu^{p} v_{\min }\right)^{2}-(\mu+1) \mu^{p-1} v_{\min } \mu^{p} v_{\min }=-\mu^{2 p-1} v_{\min }^{2}
\end{array} \quad p=1,2, \ldots, \eta\right.
$$

